\title{
Expediting in Two-Echelon Spare Parts Inventory Systems
}

\author{
Melvin Drent, Joachim Arts \\ University of Luxembourg, Luxembourg Centre for Logistics and Supply Chain Management, \\ melvin.drent@uni.lu, joachim.arts@uni.lu
}

\begin{abstract}
Problem definition: We consider dual sourcing in a distribution network for spare parts consisting of one central warehouse and multiple local warehouses. Each warehouse keeps multiple types of repairable parts to maintain several types of capital goods. The repair shop at the central warehouse has two repair options for each repairable part: a regular repair option and an expedited repair option. Irrespective of the repair option, each repairable part uses a certain resource for its repair. In the design of these inventory systems, companies need to decide upon stocking levels and expedite thresholds such that total stock investments are minimized while satisfying asset availability and expediting constraints.
\end{abstract}

Academic / Practical Relevance: Although most companies have the possibility to expedite the repair of parts in short supply, no contributions have been made that incorporate such dynamic expediting policies in repairable investment decisions. Anticipating expediting decisions that will be made later leads to substantial reductions in repairable investments.

Methodology: We use queueing theory to determine the performance of the central warehouse and subsequently find the performance of all local warehouses using binomial disaggregation. For the optimization problem, we develop a greedy heuristic and a decomposition and column generation based algorithm.

Results: Both solution approaches perform very well with average optimality gaps of 2.38 and 0.27 percent, respectively, across a large test bed of industrial size. The possibility to expedite the repair of failed parts is effective in reducing stock investments with average reductions of 7.94 percent and even reductions up to 19.61 percent relative to the state of the art.

Managerial Implications: Based on a case study at Netherlands Railways, we show how managers can significantly reduce the investment in repairable spare parts when dynamic repair policies are leveraged to prioritize repair of parts whose inventory is critically low.

Key words: inventory; spare parts; multi-item; repair; expediting; multi-echelon; column generation

History: February 6, 2018; Revisions: December 4, 2018; October 16, 2019; January 8, 2020

\section{Introduction}

For many industries and service organizations, the availability of capital goods such as rolling stock, manufacturing equipment and aircraft is of crucial importance for their operations. To ensure high availability of these capital goods, companies stock critical compo- 
nents and replace a defective component with a ready-for-use spare component after failure. Since many critical components represent a significant financial investment, defective components are usually repaired and put back on stock rather than discarded. Consequently, the availability of capital goods largely depends on the design of the underlying spare parts inventory system for repairing and supplying these so-called repairable components.

Spare parts inventory systems for capital goods often have a two-echelon structure, in which many different types of components are stocked (Cohen et al. 1997). In this paper, we study such a multi-item two-echelon spare parts inventory system. The system consists of a set of local warehouses, i.e. operating sites, that are supported by one central warehouse. Each local warehouse maintains field inventories for spare components and sends defective components to the repair shop. The repair shop repairs these defective components and sends them to the central warehouse which replenishes the local warehouses. It is obvious that next to the inventory levels of spare components, the repair operations at the repair shop affect the availability of capital goods at the operating sites. Hence, the determination of spare components inventory levels and the design of the repair operations in the repair shop are two key aspects in the design of these two-echelon spare parts inventory systems.

In the capital goods industry, it is common practice to acquire spare components together with the acquisition of the capital good because, at that time, it is possible to negotiate reasonable prices. The determination of spare components inventory levels is therefore closely related to what is known in literature as the initial spare parts supply problem (e.g., Van Houtum and Kranenburg 2015). With respect to the repair operations at the repair shop, companies often have the flexibility to expedite the repair of defective components. Although expediting comes at an extra price, either because internal repair resources are limited or because an external repair shop charges a higher price, the possibility to expedite can significantly reduce the required initial financial investment in spare parts. Indeed, expediting the repair of defective components more often implies that a smaller initial financial investment in spare parts is required to ensure the same availability of capital goods as in spare parts inventory systems where no repair flexibility is incorporated.

Hence, in the design of the spare parts inventory systems sketched in the last two paragraphs, decision makers face two major questions:

1. How many spare parts of each repairable type should the company initially purchase and place at each warehouse? 
2. When should the repair of a detective part of a given repairable type be expedited? The objective of this paper is to present a tractable optimization model that assists decision makers in answering these two questions. These questions are faced among others by Netherlands Railways (NS), the principal Dutch passenger railway operator. Our collaboration with their maintenance department led to the present work. To establish the practical value of our optimization model, we report on a case study on their data.

As is often the case in practice, we consider a setting with several capital good types (e.g. regional trains and inter-city trains; wide-body aircraft and narrow-body aircraft) and where repairables may use different repair resources (e.g. electronic and mechanical). Because not all repairs can be expedited, many companies, including NS, use agreements between the repair shop manager and the inventory manager that determine how much of the total workload can be expedited per repair resource. Hence, the objective of our optimization model is to minimize the total investment costs in spare parts while

- not exceeding a given maximum total mean number of backorders over all local warehouses for each capital good type, and

- keeping the fraction of repairs that are expedited per repair resource below a given target level. (We will also consider an alternate setting in which expediting repairs comes at additional costs rather than being constrained).

Because we consider critical components, a backorder for a spare part implies that the affected capital good becomes inoperable. Since failures of components typically occur very infrequently, a common assumption in the spare parts literature is that the probability that two or more backorders are from the same capital good at any point in time is negligible (e.g., Muckstadt 2005, Sherbrooke 2004). Under that assumption the average availability of a capital good type is the number of capital goods of that type minus the expected number of backorders of parts in that capital good type. As such, the first constraint of our optimization model guarantees a certain availability of each capital good type throughout the geographical region covered by the local warehouses.

In this paper, we provide a mathematical model for the decision problem described above. We assume that each local warehouse is replenished by an $(S-1, S)$ base stock policy. This means that each defective part is replaced with a ready-for-use item and is sent to the repair shop at the central warehouse immediately after the defect occurs. This replenishment policy is common in practice and is considered as well-suited for spare parts inventory 
control (Van Houtum and Kranenburg 2015). The central warehouse operates under an $(S, T)$ policy similar to Song and Zipkin (2009), which keeps the usual inventory position at constant level $S$, just as in a standard base stock policy. In addition, expedite threshold $T$ triggers expedited repairs when outstanding orders in the repair pipeline are too far away. This dynamic repair policy thus takes into account real-time information about the repair pipeline of the repair shop, which can be obtained through modern tracking technologies. We assume that unsatisfied demand is backordered at all warehouses. Furthermore, we assume deterministic lead times for the replenishments of the local warehouses as well as for both repair options at the central warehouse.

The main contributions of this paper are summarized as follows:

1. We are the first to integrate stocking and expedited repair decisions in multi-item two-echelon spare parts inventory systems, where parts belong to different capital good types and where parts that use the same repair resource compete for expedited repair.

2. We provide a tractable optimization model that yields a tight lower bound on the optimal solution and near optimal feasible solutions. We show that our formulation allows us to decompose the non-linear non-convex integer programming problem into sub-problems per repairable type and subsequently use column generation algorithms. For the resulting sub-problem, whose state space has dimensions equal to the number of locations plus one, we provide an efficient solution algorithm that searches over only two dimensions and where each instance involves independent Newsvendor type problems.

3. As an alternative solution approach, we provide a greedy heuristic that yields excellent results. Different from most literature on greedy heuristics in spare parts inventory systems, our greedy heuristic does not only decide upon stocking levels given a certain target service level, but also on expedite thresholds such that the fraction of the total demand that receives expedited repair per repair resource remains below a certain target level.

4. Based on a case study at NS, we present insights that will help managers to understand how a dynamic repair policy can be leveraged to reduce the total investment costs in spare parts while meeting availability targets.

In his seminal paper on the METRIC model, Sherbrooke (1968) already argued that in practice, parts in short supply should be scheduled into repair first. Though, he and most contributions on the METRIC model assume that the repair lead times of each part are i.i.d. distributed, meaning that no scheduling or prioritization in repairs is possible. 
As a direct consequence, performance obtained in practice (either investments in stock or availability) is better than theory predicts (e.g., Rustenburg 2000, Rustenburg et al. 2001), though exact percentages are lacking. In this paper, we are the first to relax this assumption by explicitly incorporating the possibility to change the repair lead time of a part based on the current state of the system, thereby actually scheduling parts in short supply into repair first. We show that effective usage of this possibility may lead to reductions in stock investments of up to 19.61 percent compared to static repair lead times. We show that such reductions remain attainable even when we incur additional costs for using this flexibility.

The remainder of this paper is organized as follows. Section 2 reviews related literature. In Section 3, we provide a formal description of the model. In Section 4, we present an exact evaluation procedure for a given control policy as well as the mathematical formulation of our decision problem. Section 5 presents two solution approaches to solve this decision problem. We show in Section 6 that these solution approaches also apply to an alternate setting in which expediting has an additional cost. Section 7 provides managerial insights based on a case study at NS and evaluates the performance of both solution approaches in a large test bed. Finally, some concluding remarks are presented in Section 8.

\section{Literature review}

Although spare parts inventory systems have been studied extensively in a variety of settings, our review involves literature with similar modeling assumptions or similar solution approaches as those used in this paper. For an extensive discussion of the existing literature in the broad field of spare parts inventory management, we refer the reader to Basten and Van Houtum (2014), Van Houtum and Kranenburg (2015) and Muckstadt (2005).

This paper contributes to the classical research line of multi-item spare parts inventory systems that started in 1968 with the seminal paper of Sherbrooke on the METRIC model. This model assumes that demand follows a Poisson process and that all warehouses operate under base stock policies. Via an approximative evaluation method, expected backorders at all local warehouses are determined for a given control policy. Since then, many extensions have been made to the METRIC model: While some researchers have focused on deriving exact steady state distributions (e.g., Graves 1985, Simon 1971), others have extended the model itself by integrating hierarchical or indentured parts structures (e.g., Muckstadt 1973), by allowing for part failures that lead to downtime after a delay (e.g., Bitton et al. 
2019), or by including emergency shipments (e.g., Alfredsson and Verrijdt 1999, Lee 1987, Howard et al. 2015). The exact evaluation procedure of our paper shows similarities with Graves (1985). The main difference is that he considers only one supply mode at the central warehouse, whereas we consider both a regular and an expedited supply mode.

The system studied in this paper extends previous research which examined inventory models with multiple supply modes. We refer to Svoboda et al. (2019) for an extensive discussion of such inventory models, here we discuss only the important and more relevant results. Since optimal control policies for inventory systems with expediting have complex structures (e.g., Feng et al. 2006, Whittemore and Saunders 1977), most recent papers study relatively simple heuristic policies and aim at finding (near) optimal parameters.

For single-echelon inventory systems under periodic review, an often studied heuristic policy is the dual-index policy, in which two different inventory positions are kept track off: The inventory position including arrivals within the expedited lead time and the inventory position including arrivals within the regular lead time (e.g., Arts et al. 2011, Sheopuri et al. 2010, Sun and Van Mieghem 2019, Veeraraghavan and Scheller-Wolf 2008). Moinzadeh and Schmidt (1991) consider a similar policy for single-echelon inventory systems facing Poisson under continuous review. They focus on obtaining performance measures for a given dual-index policy when both the expedited and regular lead time are deterministic. Song and Zipkin (2009) reinterpret and extend the work of Moinzadeh and Schmidt (1991) by showing that the same inventory system with a dual-index policy and stochastic lead times is a special type of product form queueing network with one or more overflow bypasses. The dual-index policy in the setting of Moinzadeh and Schmidt (1991) and Song and Zipkin (2009) is in fact optimal for the special case where the regular repair lead time has a shifted exponential distribution and the base stock level for the regular inventory position is fixed (Arts et al. 2016). The policy that we consider for the central warehouse is equivalent to the dual-index policy of Song and Zipkin (2009). The methods of Song and Zipkin (2009) have been incorporated in a two-echelon spare parts inventory system before, albeit to decide upon emergency shipments from a so-called support warehouse to the local warehouses (Howard et al. 2015).

Literature on multiple supply modes in multi-echelon distribution systems is relatively scarce. Building upon the dual-index policy of Moinzadeh and Schmidt (1991), Moinzadeh and Aggarwal (1997) consider a two-echelon distribution system facing Poisson demand 
under continuous review in which all warehouses have the option to replenish their inventory through an expedited or regular supply channel. Similar to Moinzadeh and Schmidt (1991), they assume deterministic lead times for both types of shipments to all warehouses. Moinzadeh and Aggarwal (1997) describe a procedure to find optimal policy parameters and show that this system substantially improves its single-sourcing counterpart. By contrast to their paper, we consider a system where only the central warehouse has two supply modes. Yet, we impose no limitations on the lead times of those supply modes.

Within the stream of literature focusing on inventory systems for repairable items, many contributions have been made on either expediting the repair or prioritizing the scheduling of repairs in the repair shop. As deriving structural properties of optimal policies is known to be complex when the number of different repairable types increases (Tiemessen and Van Houtum 2013), most contributions in this area resort to heuristic priority rules. We distinguish two categories of such heuristic priority rules. Under static priority rules, the priority of a repairable depends on its type only. Although these type of priority rules are relatively simple, several studies have shown that such rules outperform simple first come first serve rules in terms of investment costs (e.g., Adan et al. 2009, Sleptchenko et al. 2005). Under more sophisticated dynamic priority rules, the priority of a repairable also depends on the current state of the system. The expediting policy in our model falls into this latter category as it essentially changes the repair lead time of a part based on the current state of the repair pipeline. In a recent contribution, Arts et al. (2016) study an expediting policy similar to the present model, albeit in a single-echelon single-item setting under fluctuating demand. They remark that this expediting policy does not suffer from the tractability issues that other dynamic priority rules suffer from, while still providing the lead time flexibility inherent to this category of heuristic priority rules. Loeffen (2012) shows that this expediting policy can also be implemented in elaborate simulation models of the repair shop and yield similar performance.

Few researchers have considered dynamic repair priority rules in multi-echelon inventory systems for repairable items. Pyke (1990) jointly addresses dynamic repair and inventory allocation decisions in a two-echelon system very similar to the one we study. He sketches a mathematical formulation of the problem to emphasize its complexity and computational intractability and subsequently resorts to simulation experiments. More recently, Caggiano et al. (2006) consider a similar problem related to dynamic repair and inventory allocation 
decisions. Different from the present work, their model is a finite-horizon, periodic-review model involving only one repair resource focusing on operational decisions for repairable spare parts in the exploitation phase of capital goods.

On the analysis side, we use two techniques that are widely used in the context of multiitem spare parts inventory optimization. The first technique, decomposition and column generation, is appropriate for problems that have a complicated aggregation constraint that links the different repairable types. Decomposing this problem leads to relatively simple sub-problems per repairable type. This technique has been used extensively in recent contributions on spare part inventory optimization (e.g., Alvarez et al. 2013, 2015, Arts 2017, Kranenburg and Van Houtum 2007, Topan et al. 2017, Wong et al. 2007). Most contributions only consider an aggregated service level constraint that links the different repairable types. In this paper, repairable types are not only linked through such a service level constraint, but also through the maximally allowed mean fraction of expedited repairs over all repairable types that use the same repair resource. Arts (2017) considers a similar optimization model with linking constraints on both expedited repairs and service levels. The major difference between our work and Arts (2017) is that we consider a two-echelon spare parts inventory system. For an extensive discussion on decomposition and column generation, we refer to Dantzig and Wolfe (1960) and Lübbecke and Desrosiers (2005).

The second technique, a greedy method, is a search algorithm that iteratively selects the

alternative that has the highest ratio of improvement in performance over cost increase until a feasible solution is obtained. A greedy method is quick, intuitive, easy to implement and provides satisfactory results. Although the technique has been applied in many papers on multi-item spare parts inventory optimization (e.g., Cohen et al. 1990, Kranenburg and Van Houtum 2009, Topan et al. 2017, Wong et al. 2007), none have proposed a greedy method on both stocking and expediting decisions that yields good results.

\section{Model description}

In this section, we first provide a brief description of the two-echelon spare parts inventory system and introduce the notation that we use throughout this paper. We then describe the policy we propose to control the system.

\subsection{Description and notation}

We consider a two-echelon spare parts inventory system consisting of a central warehouse and multiple local warehouses. Let the non-empty set of local warehouses be denoted 
by $N_{l}$. The set of all warehouses is denoted by $N$, i.e. $N=\{0\} \cup N_{l}$. Hence, the central warehouse has index zero while the local warehouses are numbered as $n=1,2, \ldots,\left|N_{l}\right|$. Each local warehouse $n$ is responsible for serving an operating site consisting of a number of capital goods, which may be of the same or different type. Let $C$ denote the nonempty set of capital good types. Each capital good type $c \in C$ consists of a number of critical components that fail infrequently and independently. These critical components are crucial for operating the capital good, i.e. the capital good is down if one of these components fails. The components are at such levels in the material breakdown structure of the capital good that they can be replaced as a whole by spare parts. Component types are also called Stock Keeping Units (SKUs). Let $M$ denote the non-empty set of critical SKUs that occur in the configurations of the different capital good types. The SKUs are numbered as $m=1,2, \ldots,|M|$ and each part of SKU $m \in M$ has an acquisition $\operatorname{cost} c_{a}^{m}$. The set of SKUs that occur in the configuration of capital good type $c \in C$ is denoted by $M_{c}^{C}$. There is a set of repair resources, denoted by $R$, that are used to repair failed parts in the repair shop (at the central warehouse). The SKUs that use repair resource $r \in R$ in their repair are contained in the set $M_{r}^{R}$. We assume that $M_{c}^{C}$ and $M_{r}^{R}$ partition $M$, i.e. $\cup_{c \in C} M_{c}^{C}=\cup_{r \in R} M_{r}^{R}=M$ and $\cap_{c \in C} M_{c}^{C}=\cap_{r \in R} M_{r}^{R}=\emptyset$. This assumption is common in practice and simplifies notation considerably; it is however not essential to our analysis. We briefly show in Online Appendix D how this assumption can be readily relaxed along similar lines as is done in Kranenburg and Van Houtum (2007).

Demand for SKU $m \in M$ at local warehouse $n \in N_{l}$ is a Poisson process with rate $\lambda_{m, n}$. This demand model is common in literature and accurate in practice for spare parts (e.g., Graves 1985). When a demand for SKU $m$ occurs at local warehouse $n$, it will be filled from stock, or backordered if the stock is depleted. In the latter case, the capital good remains down until a spare part becomes available at the local warehouse. The failed part is shipped to the repair shop at the central warehouse, where all failed parts are immediately sent into regular or expedited repair, where the corresponding resource $r \in R$ is used for repair. At the same time, the central warehouse ships a spare part to the local warehouse from its inventory, if it has an available spare part. Otherwise, the replenishment order is backordered at the central warehouse until a part is repaired and becomes available. Upon completion of repair a part is put back on stock at the central warehouse. 
The order and shipment time for a spare part of SKU $m$ from the central warehouse to local warehouse $n$ is fixed and denoted by $t_{m, n}$. Note that $t_{m, n}$ excludes any waiting time at the central depot when a spare part is not available. For returned failed parts at the repair shop, it takes either $t_{m, 0}^{r e g}$ time units, in case of the regular repair, or $t_{m, 0}^{\text {exp }}$ time units, in case of the expedited repair, until the part is returned to the spare parts stock at the central warehouse. We assume that both repair times are fixed, with $t_{m, 0}^{r e g}>t_{m, 0}^{e x p}>0$. Figure 1 provides a graphical representation of the system under consideration and notation is summarized in Table 1 (including notation introduced later).

Figure 1 Two-echelon spare parts inventory system with expediting

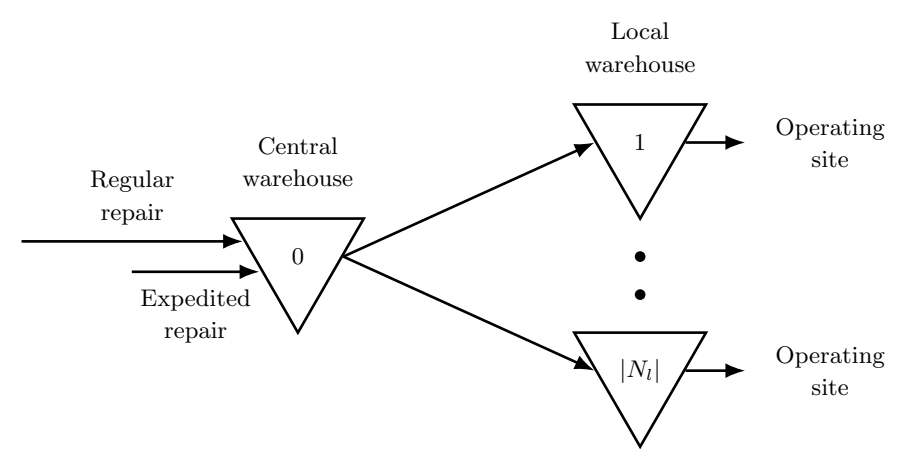

\subsection{Control policy}

Each failed part at a local warehouse results in an immediate replenishment order at the central warehouse. This implies that the inventory positions of a given SKU $m \in M$ remain constant at all local warehouses. Hence, we have base stock control at each local warehouse $n \in N_{l}$ for each SKU $m \in M$ and we denote the corresponding base stock levels by $S_{m, n}$.

The central warehouse is controlled by a dual-index policy. This policy has two parameters for each SKU $m \in M$, integers $S_{m, 0}$ and $S_{m, 0}^{\prime}$, with $S_{m, 0} \geq S_{m, 0}^{\prime}$. Let $t_{m, 0}^{1}=t_{m, 0}^{r e g}-t_{m, 0}^{e x p}$, i.e. the additional regular lead time, and $t_{m, 0}^{2}=t_{m, 0}^{e x p}$. We define two inventory positions for each SKU $m: I P_{m, 0}^{1}$ and $I P_{m, 0}^{2} I P_{m, 0}^{1}$ is the usual local inventory position and includes net inventory $I N_{m, 0}$ (on-hand stock $O H_{m, 0}$ minus any backorders $B O_{m, 0}$ ) plus all parts in repair $X_{m, 0} . I P_{m, 0}^{2}$ is similar but only includes those parts in repair $X_{m, 0}^{2}$ that will be repaired and returned to on-hand stock within $t_{m, 0}^{2}$ time units. Hence, the number of parts in repair that will not be repaired and returned to on-hand stock within $t_{m, 0}^{2}$ time units $X_{m, 0}^{1}$ is equal to $X_{m, 0}-X_{m, 0}^{2}$. Figure 2 provides a graphical representation of the two different inventory positions at the central warehouse. 
Table 1 Overview of notation

\begin{tabular}{|c|c|}
\hline Notation & Description \\
\hline \multicolumn{2}{|l|}{ Sets } \\
\hline$N$ & Set of all warehouses. \\
\hline$N_{l} \subset N$ & Set of local warehouses. \\
\hline$M$ & Set of all SKUs. \\
\hline$C$ & Set of capital good types. \\
\hline$R$ & Set of repair resources. \\
\hline$M_{r}^{R} \subseteq M$ & Set of SKUs that use repair resource $r \in R$ in the repair of failed parts. \\
\hline$M_{c}^{C} \subseteq M$ & Set of SKUs that occur in the configuration of capital good type $c \in C$. \\
\hline \multicolumn{2}{|r|}{ 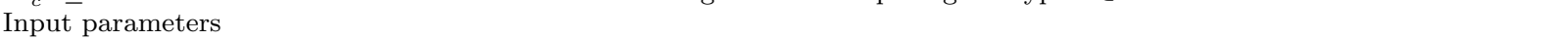 } \\
\hline$\lambda_{m, n}$ & Demand intensity for SKU $m \in M$ at warehouse $n \in N$ \\
\hline$\delta_{m}^{r}$ & Fraction of demands over all parts of SKUs $k \in M_{r}^{R}$ that are from SKU $m$, i.e. $\frac{\lambda_{m, 0}}{\sum_{k \in M_{r}^{R}} \lambda_{k, 0}}$ \\
\hline$t_{m, n}$ & Lead time from the central warehouse to local warehouse $n \in N_{l}$ of SKU $m \in M$ \\
\hline$t_{m, 0}^{r e g}$ & Regular repair lead time of SKU $m \in M$ \\
\hline$t_{m, 0}^{e x p}$ & Expedited repair lead time of SKU $m \in M$, also denoted by $t_{m, 0}^{2}$. \\
\hline$t_{m, 0}^{1}$ & Additional regular repair lead time of SKU $m \in M$ \\
\hline$c_{a}^{m}$ & Acquisition cost for SKU $m \in M$. \\
\hline$\tau$ & Useful lifespan of each SKU $m \in M$ \\
\hline$\kappa$ & Expediting cost multiplier for each SKU $m \in M$ \\
\hline$c_{e}^{m}$ & Cost for expediting the repair of SKU $m \in M$, i.e. $\kappa \cdot c_{a}^{m}$. \\
\hline$c_{d}^{m}$ & Depreciation cost rate for SKU $m \in M$, i.e. $c_{a}^{m} / \tau$ \\
\hline $\mathcal{B}_{c}^{\max }$ & The maximally allowed mean number of backorders over all SKUs $m \in M_{c}^{C}$ for capital good type $c \in C$. \\
\hline $\mathcal{E}_{r}^{\max }$ & $\begin{array}{l}\text { The maximally allowed mean fraction of expedited repairs over all SKUs } m \in M_{r}^{R} \text { that use repair resource } \\
r \in R \text { during their repair. }\end{array}$ \\
\hline \multicolumn{2}{|r|}{ 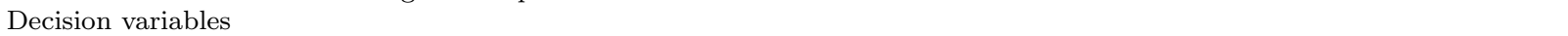 } \\
\hline$S_{m, n}$ & Base stock level of SKU $m \in M$ at warehouse $n \in N$. \\
\hline $\mathbf{S}_{m}$ & The vector $\left(S_{m, 0}, S_{m, 1}, \ldots, S_{m,\left|N_{l}\right|}\right)$ \\
\hline $\mathbf{S}$ & The base stock levels matrix $\left[S_{m, n}\right]$. \\
\hline$T_{m}$ & Expedite threshold of SKU $m \in M$. \\
\hline$T$ & The vector $\left(T_{1}, T_{2}, \ldots, T_{|M|}\right)$ \\
\hline \multicolumn{2}{|l|}{ State variables } \\
\hline$X_{m, 0}$ & Number of outstanding repairs of SKU $m \in M$ at the central warehouse. \\
\hline$X_{m, 0}^{2}$ & $\begin{array}{l}\text { Number of outstanding repairs of SKU } m \in M \text { at the central warehouse that will be repaired within } t_{m, 0}^{2} \\
\text { time units. }\end{array}$ \\
\hline$X_{m, 0}^{1}$ & $\begin{array}{l}\text { Number of outstanding repairs of SKU } m \in M \text { at the central warehouse that will not be repaired within } \\
t_{m, 0}^{2} \text { time units, i.e. } X_{m, 0}-X_{m, 0}^{2}\end{array}$ \\
\hline$X_{m, n}$ & Number of outstanding orders of SKU $m \in M$ at local warehouse $n \in N_{l}$ \\
\hline \multicolumn{2}{|r|}{ 260. } \\
\hline$E B O_{m, n}\left(\mathbf{S}_{m}, T_{m}\right)$ & $\begin{array}{l}\text { Mean number of backorders for SKU } m \text { at local warehouse } n \in N_{l} \text { under a given control policy }\left(\mathbf{S}_{m}, T_{m}\right) \text {, } \\
\text { i.e. } \sum_{x=S_{m, n}+1}^{\infty}\left(x-S_{m, n}\right) \mathbb{P}\left\{X_{m, n}=x\right\} \text {. }\end{array}$ \\
\hline$E B O_{c}(\mathbf{S}, T)$ & $\begin{array}{l}\text { Aggregate mean number of backorders for capital good type } c \in C \text { under a given control policy }(\mathbf{S}, T) \text {, } \\
\text { i.e. } \sum_{m \in M_{c}^{C}} \sum_{n \in N_{l}} E B O_{m, n}\left(\mathbf{S}_{m}, T_{m}\right) \text {. }\end{array}$ \\
\hline$E X P_{m}\left(T_{m}\right)$ & $\begin{array}{l}\text { Fraction of failed parts of SKU } m \in M \text { that utilize the expedited repair option under a given expedite } \\
\text { threshold }\left(T_{m}\right) \text {, i.e. } \mathbb{P}\left\{X_{m, 0}^{1}=T_{m}\right\} \text {. }\end{array}$ \\
\hline$E X P_{r}(T)$ & $\begin{array}{l}\text { Aggregate mean fraction of failed parts over all SKUs } m \in M_{r}^{R} \text { that utilize the expedited repair option } \\
\text { under a given expedite threshold vector } T \text {, i.e. } \sum_{m \in M_{r}^{R}} \delta_{m}^{r} E X_{m}\left(T_{m}\right) .\end{array}$ \\
\hline$C(\mathbf{S})$ & $\begin{array}{l}\text { The total investment costs in spare parts under a given base stock levels matrix } \mathbf{S} \text { i.e. } \\
\sum_{m \in M} \sum_{n \in N} c_{a}^{m} S_{m, n} \text {. }\end{array}$ \\
\hline$C_{d}(\mathbf{S})$ & $\begin{array}{l}\text { The total depreciation cost rate in spare parts under a given base stock levels matrix } \mathbf{S} \text {, i.e. } \\
\sum_{m \in M} \sum_{n \in N} c_{d}^{m} S_{m, n} \text {. }\end{array}$ \\
\hline$C_{e}(T)$ & $\begin{array}{l}\text { The total repair expediting cost rate under a given expedite threshold vector } T \text {, i.e. } \\
\sum_{m \in M} c_{e}^{m} \lambda_{m, 0} E X P_{m}\left(T_{m}\right) \text {. }\end{array}$ \\
\hline$C_{P}^{U B}\left(C_{P}^{L B}\right)$ & Upper (lower) bound for the optimal solution to problem $(P)$ \\
\hline$C_{B M}^{L B}$ & Lower bound for the optimal solution of a benchmark instance $B M$. \\
\hline
\end{tabular}

The dual-index policy works as follows: Keep $I P_{m, 0}^{1}$ at constant level $S_{m, 0}$ (as in standard base stock control) and also $I P_{m, 0}^{2} \geq S_{m, 0}^{\prime}$. Thus upon the demand of a part and the return of a failed part of SKU $m \in M$, we first examine $I P_{m, 0}^{2}$. If $I P_{m, 0}^{2}$ (after the failed part is returned, but before deciding upon the repair option) is already $S_{m, 0}^{\prime}$ or greater, we send it 
Figure 2 Inventory positions at the central warehouse

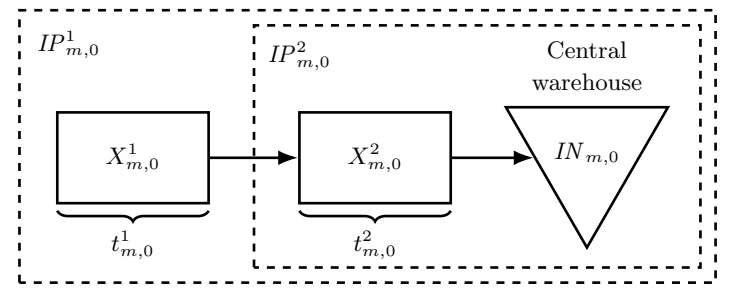

into regular repair. However, if a regular repair would leave $I P_{m, 0}^{2}<S_{m, 0}^{\prime}$, then we use the expedited repair option. Note that $I P_{m, 0}^{2}=I N_{m, 0}+X_{m, 0}^{2}=I P_{m, 0}^{1}-X_{m, 0}^{1}=S_{m, 0}-X_{m, 0}^{1}$, and thus, equivalently, the dual-index policy keeps $S_{m, 0}-X_{m, 0}^{1} \geq S_{m, 0}^{\prime}$. Hence, defining expedite threshold $T_{m}=S_{m, 0}-S_{m, 0}^{\prime} \forall m \in M$, the dual-index policy sends failed parts into regular repair as long as $X_{m, 0}^{1} \leq T_{m}$ (cf. Song and Zipkin 2009).

Let $\mathbf{S}_{m}=\left(S_{m, 0}, S_{m, 1}, \ldots, S_{m,\left|N_{l}\right|}\right), m \in M$, denote the vector of base stock levels for SKU $m$. Then, a control policy $(\mathbf{S}, T)$ is denoted by base stock levels matrix $\mathbf{S}$ and a vector $T=\left(T_{1}, T_{2}, \ldots, T_{|M|}\right)$ containing the expedite thresholds of each SKU $m \in M$.

\section{Performance evaluation and problem formulation}

In this section, we provide an exact evaluation procedure for a given control policy $(\mathbf{S}, T)$, and we present the mathematical formulation of the decision problem.

\subsection{Exact evaluation of a given control policy}

The evaluation of a given control policy $(\mathbf{S}, T)$ can be done per SKU. Consider therefore some SKU $m \in M$ that has base stock vector $\mathbf{S}_{m}$ and expedite threshold $T_{m}$. We first consider the performance of SKU $m$ at the central warehouse, and subsequently link this to its performance at all local warehouses.

Key in evaluating the performance of the central warehouse for SKU $m$ is to obtain the distribution of the number of parts in repair $X_{m, 0}$. Since each failure of SKU $m$ results in an immediate replenishment request for SKU $m$ at the central warehouse, the demand process for parts of SKU $m$ as seen by the central warehouse is a Poisson process with constant rate $\lambda_{m, 0}=\sum_{n \in N_{l}} \lambda_{m, n}$. Each replenishment request for SKU $m$ is accompanied by a failed part that goes into repair. Hence, failed parts of SKU $m$ enter the repair pipeline according to a Poisson process with constant rate $\lambda_{m, 0}$. The fraction of demands for SKU $m$ over demands from all SKUs that use the same repair resource $r \in R$ as SKU $m$ uses, is then given by $\delta_{m}^{r}=\frac{\lambda_{m, 0}}{\sum_{k \in M_{r}^{R}} \lambda_{k, 0}}$. Now, under the dual-index policy described in the previous 
section, the repair pipeline of each SKU $m$ can be seen as an open queueing network with outside Poisson arrivals at constant rate $\lambda_{m, 0}$ and two $\bullet / D / \infty$ queues that cause delays of $t_{m}^{1}$ and $t_{m}^{2}$ time units (see Figure 3 ).

\section{Figure 3 Repair pipeline as an open queueing network}

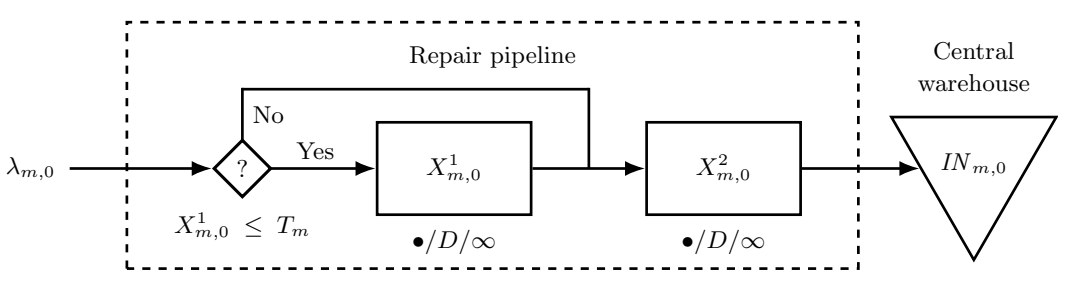

Observe that a normal repair first passes through queue 1, where it remains $t_{m}^{1}$ units, and then passes through queue 2 , where it remains $t_{m}^{2}$ units. Failed parts that receive expedited repair bypass the first part of the repair pipeline and only pass through queue 2. After a part completes queue 2 , it arrives to inventory at the central warehouse. In effect, the dual-index policy directs failed parts of SKU $m$ into normal repair as long as the number of repairs at queue 1, i.e. $X_{m, 0}^{1}$, is not greater than the expedite threshold $T_{m}$. When an arriving failed part would overflow $T_{m}$, the failed part bypasses queue 1 and goes directly to queue 2, that is, the failed part goes into expedited repair. In the queueing literature, this is sometimes referred to as jump over blocking or as an overflow bypass (e.g., Lam 1977, Song and Zipkin 2009).

The distribution of the number of parts in repair $X_{m, 0}$, follows from the joint distribution of $\left(X_{m, 0}^{1}, X_{m, 0}^{2}\right)$. Let $p_{m}\left(x_{1}, x_{2}\right)=\mathbb{P}\left\{X_{m, 0}^{1}=x_{1}, X_{m, 0}^{2}=x_{2}\right\}$ denote the steadystate joint distribution of $\left(X_{m, 0}^{1}, X_{m, 0}^{2}\right)$. Let $\phi_{m}^{1}\left(x_{1}\right)$ and $\phi_{m}^{2}\left(x_{2}\right)$ denote the Poisson probabilities $e^{\lambda_{m, 0} \cdot t_{m}^{1}}\left(\lambda_{m, 0} \cdot t_{m}^{1}\right)^{x_{1}} / x_{1}$ ! and $e^{\lambda_{m, 0} \cdot t_{m}^{2}}\left(\lambda_{m, 0} \cdot t_{m}^{2}\right)^{x_{2}} / x_{2}$ !, respectively. The support of $\left(X_{m, 0}^{1}, X_{m, 0}^{2}\right)$ is denoted by $\mathscr{X}\left(T_{m}\right)=\left\{\left(x_{1}, x_{2}\right) \in \mathbb{N}_{0}^{2}: x_{1} \leq T_{m}\right\}$. Then, as shown by Lam (1977) and Song and Zipkin (2009), the joint distribution of $\left(X_{m, 0}^{1}, X_{m, 0}^{2}\right)$ has product-form

$$
p_{m}\left(x_{1}, x_{2}\right)=\frac{\phi_{m}^{1}\left(x_{1}\right) \phi_{m}^{2}\left(x_{2}\right)}{\sum_{x_{1} \leq T_{m}} \phi_{m}^{1}\left(x_{1}\right)}, \quad\left(x_{1}, x_{2}\right) \in \mathscr{X}\left(T_{m}\right) .
$$

Letting $p_{m}(x)=\mathbb{P}\left\{X_{m, 0}=x\right\}$ denote the equilibrium probability of the number of parts in repair $X_{m, 0}$ and $\phi_{m}(x)$ denote the Poisson probability $e^{\lambda_{m, 0} \cdot t_{m}^{r e g}}\left(\lambda_{m, 0} \cdot t_{m}^{r e g}\right)^{x} / x$, we obtain

$$
p_{m}(x)=\sum_{i=0}^{x} p_{m}(i, x-i)=\left(\sum_{x_{1} \leq T_{m}} \phi_{m}^{1}\left(x_{1}\right)\right)^{-1} \begin{cases}\phi_{m}(x) & x \leq T_{m} \\ \sum_{i=0}^{T_{m}} \phi_{m}^{1}(i) \phi_{m}^{2}(x-i) & x>T_{m} .\end{cases}
$$


The main performance measures of the central warehouse are now easily obtained. In particular, the number of backorders $B O_{m, 0}$ for SKU $m$ is equal to $\left(X_{m, 0}-S_{m, 0}\right)^{+}$, where $x^{+}=\max (0, x)$. For its probability distribution, we have

$$
\mathbb{P}\left\{B O_{m, 0}=x\right\}= \begin{cases}\sum_{j=0}^{S_{m, 0}} p_{m}(j), & x=0, \\ p_{m}\left(S_{m, 0}+x\right), & x>0 .\end{cases}
$$

In addition, letting $\rho_{m}=\lambda_{m, 0} \cdot t_{m, 0}^{1}$, the fraction of failed parts of SKU $m$ that utilize the expedite repair option is given by

$$
\operatorname{EXP}_{m}\left(T_{m}\right)=\mathbb{P}\left\{X_{m, 0}^{1}=T_{m}\right\}=\frac{\rho_{m}^{T_{m}}}{T_{m} !}\left(\sum_{i=0}^{T_{m}} \frac{\rho_{m}^{i}}{i !}\right)^{-1}
$$

which is the (Erlang) blocking probability of an $M / G / c / c$ queue, where the numbers of parallel servers $c$ is equal to expedite threshold $T_{m}$ (e.g., Gross et al. 2008).

Key in evaluating the performance of each local warehouse $n \in N_{l}$ for SKU $m$ is to obtain the distribution of orders outstanding for each local warehouse. Therefore we need to determine the distribution of backorders for a SKU $m$ at the central warehouse that belong to local warehouse $n$.

Simon (1971) shows that when outstanding orders at the central warehouse are filled on a first-come first-served bases, then each backorder at the central warehouse belongs to local warehouse $n$ with probability $\frac{\lambda_{m, n}}{\lambda_{m, 0}}$, independently across backorders. Let $B O_{m, 0}^{n}$ denote the number of backorders of local warehouse $n$ in the backorder queue of SKU $m$ at the central warehouse. Then, by conditioning on the number of backorders of SKU $m$ at the central warehouse and using Simon's result that the conditional distribution of $B O_{m, 0}^{n}$ is a binomial distribution, we obtain the following probability distribution for this number of backorders

$$
\begin{aligned}
\mathbb{P}\left\{B O_{m, 0}^{n}=x\right\} & =\sum_{y=x}^{\infty} \mathbb{P}\left\{B O_{m, 0}^{n}=x \mid B O_{m, 0}=y\right\} \mathbb{P}\left\{B O_{m, 0}=y\right\} \\
& =\sum_{y=x}^{\infty}\left(\begin{array}{c}
y \\
x
\end{array}\right)\left(\frac{\lambda_{m, n}}{\lambda_{m, 0}}\right)^{x}\left(1-\frac{\lambda_{m, n}}{\lambda_{m, 0}}\right)^{y-x} \mathbb{P}\left\{B O_{m, 0}=y\right\} .
\end{aligned}
$$

Now, we determine the distribution of the outstanding orders of SKU $m$ at each local warehouse $n$. The outstanding orders at any time $t$ consists of demand that occurred in the interval $\left(t-t_{m, n}, t\right)$ (notation $\left.D_{m, n}\left(t-t_{m, n}, t\right)\right)$ and backorders at the central warehouse that 
belong to local warehouse $n$ at time $t-t_{m, n}$ (notation $\left.B O_{m, 0}^{n}(t)\right)$, i.e. $X_{m, n}(t)=D_{m, n}(t-$ $\left.t_{m, n}, t\right)+B O_{m, 0}^{n}(t)$. Since the Poisson process has independent increments, $D_{m, n}\left(t-t_{m, n}, t\right)$ and $B O_{m, 0}^{n}(t)$ are independent random variables so that in stationary state

$$
X_{m, n}=D_{m, n}+B O_{m, 0}^{n},
$$

where $D_{m, n}$ has a Poisson distribution with mean $\lambda_{m, n} t_{m, n}$ and the distribution of $B O_{m, 0}^{n}$ is given in (1). Therefore the stationary distribution of $X_{m, n}$ is obtained by convolution.

From this point, the main performance measure of each local warehouse is easily obtained. For SKU $m$, the number of backorders $B O_{m, n}$ at local warehouse $n$ is equal to $\left(X_{m, n}-S_{m, n}\right)^{+}$. Its probability distribution is then obtained in a similar way as the probability distribution of the number of backorders at the central warehouse. In particular, the mean number of backorders for SKU $m$ at local warehouse $n$ is given by

$$
E B O_{m, n}\left(\mathbf{S}_{m}, T_{m}\right)=\sum_{x=S_{m, n}+1}^{\infty}\left(x-S_{m, n}\right) \mathbb{P}\left\{X_{m, n}=x\right\} .
$$

\subsection{Problem formulation}

For a given control policy $(\mathbf{S}, T)$, we define the total investment costs in spare parts as

$$
C(\mathbf{S})=\sum_{m \in M} \sum_{n \in N} c_{a}^{m} S_{m, n},
$$

the aggregate mean number of backorders for capital good type $c$ as

$$
E B O_{c}(\mathbf{S}, T)=\sum_{m \in M_{c}^{C}} \sum_{n \in N_{l}} E B O_{m, n}\left(\mathbf{S}_{m}, T_{m}\right),
$$

and the aggregate mean fraction of failed parts of all SKUs $m \in M_{r}^{R}$ that utilize the expedited repair option using repair resource $r \in R$ as

$$
\operatorname{EXP}_{r}(T)=\sum_{m \in M_{r}^{R}} \delta_{m}^{r} E X P_{m}\left(T_{m}\right)
$$

The objective of our decision problem is to minimize the total investment costs in spare parts while keeping the mean number of aggregate backorders for each capital good type $c \in C$ below $\mathcal{B}_{c}^{\max }$ and keeping the fraction of repairs that are expedited per repair resource $r \in R$ below $\mathcal{E}_{r}^{\max }$. Combining the aforementioned results in the following mathematical formulation of our decision problem which we call problem $(P)$ :

$$
\min _{\{\mathbf{S}, T\}} \quad C(\mathbf{S})
$$




$$
\begin{array}{lll}
\text { subject to } & E \mathcal{O}_{c}(\mathbf{S}, T) \leq \mathcal{B}_{c}^{\max }, & \forall c \in C \\
& \operatorname{EXP}_{r}(T) \leq \mathcal{E}_{r}^{\text {max }}, & \forall r \in R \\
& \mathbf{S} \in \mathscr{S}, \quad T \in \mathbb{N}_{0}^{|M|}, &
\end{array}
$$

where $\mathscr{S}=\left\{\mathbf{S}: S_{m, n} \in \mathbb{N}_{0}, \forall m \in M\right.$ and $\left.\forall n \in N\right\}$. Let $\left(\mathbf{S}^{*}, T^{*}\right)$ denote an optimal solution to problem $(P)$ and let $C_{P}$ be the corresponding optimal cost.

We note that problem $(P)$ can be considered as a non-linear non-convex knapsack problem with multiple constraints, where more than one copy of each item can be selected. It is well-known that even the simplest type of knapsack problems belongs to the class of $\mathcal{N} \mathcal{P}$-hard problems (Kellerer et al. 2004). As our knapsack problem is more complex, it is very likely that also for problem $(P)$ no polynomial time optimization algorithm exists.

\section{Optimization of base stock levels and expedite thresholds}

The focus of this section is on finding the optimal base stock levels and expedite thresholds. We first present a decomposition and column generation (DCG) algorithm to construct a lower bound for problem $(P)$. We then show how the sub-problem of this algorithm can be solved efficiently. We continue with showing how to find a good feasible solution for problem $(P)$. We conclude this section with devising an alternative solution approach in which we greedily optimize the expedite thresholds and base stock levels.

\subsection{Constructing lower bounds}

We first reformulate problem $(P)$ as a partitioning problem so that we can apply the technique of column generation (also known as Dantzig-Wolfe decomposition). This technique was pioneered by Dantzig and Wolfe (1960) and a thorough modern treatment is given by Lübbecke and Desrosiers (2005). Thus we obtain an integer linear program for which we relax the integrality constraints. We refer to this problem as the master problem $(M P)$. Let $K_{m}$ be the set of all policies $k$ for SKU $m \in M$ that respect constraint (6) of problem $(P)$. Each policy $k \in K_{m}$ has base stock vector $\mathbf{S}_{m}^{k}:=\left(S_{m, 0}^{k}, S_{m, 1}^{k}, \ldots, S_{m,\left|N_{l}\right|}^{k}\right)$ and expedite threshold $T_{m}^{k}$. Let $x_{m}^{k} \in\{0,1\}, m \in M, k \in K_{m}$, denote the decision variable indicating whether policy $k$ is chosen $\left(x_{m}^{k}=1\right)$ for SKU $m$ or not $\left(x_{m}^{k}=0\right)$. Then, by relaxing the integrality constraint on $x_{m}^{k}$, the master problem $(M P)$ is defined as follows:

$$
(M P) \min _{\left\{x_{m}^{k}: m \in M, k \in K_{m}\right\}} \sum_{m \in M} \sum_{n \in N} \sum_{k \in K_{m}} c_{a}^{m} S_{m, n}^{k} x_{m}^{k}
$$




$$
\begin{aligned}
& \text { subject to } \quad \sum_{m \in M_{c}^{C}} \sum_{n \in N_{l}} \sum_{k \in K_{m}} E B O_{m, n}\left(\mathbf{S}_{m}^{k}, T_{m}^{k}\right) x_{m}^{k} \leq \mathcal{B}_{c}^{\max }, \quad \forall c \in C \\
& \sum_{m \in M_{r}^{R}} \sum_{k \in K_{m}} \delta_{m}^{r} E X P_{m}\left(T_{m}^{k}\right) x_{m}^{k} \leq \mathcal{E}_{r}^{\max }, \quad \forall r \in R \\
& \sum_{k \in K_{m}} x_{m}^{k}=1, \quad \forall m \in M \\
& x_{m}^{k} \geq 0, \quad \forall m \in M, \forall k \in K_{m}
\end{aligned}
$$

Let $C_{P}^{L B}$ denote the optimal cost for master problem $(M P)$. Due to the relaxation of the integrality constraint on $x_{m}^{k}$, an optimal cost $C_{P}^{L B}$ is also a lower bound on the optimal cost for problem $(P), C_{P}$.

Since the set $K_{m}$ contains an infinite number of policies, a restricted master problem $(R M P)$ is introduced in which, for each SKU $m \in M$, only a small subset of policies $K_{m}^{r e s} \subseteq$ $K_{m}$ is considered. After solving $(R M P)$ to optimality, we are interested in policies $K_{m} \backslash K_{m}^{r e s}$ that will improve the solution of $(R M P)$ if they are added. To check whether such policies exist, we solve, for each SKU $m$, a column generation sub-problem. To this end, let $p_{c}$ denote the dual variable of $(R M P)$ corresponding with the expected backorder constraint (8) for capital good type $c \in C$, let $\rho_{r}$ denote the dual variable of $(R M P)$ corresponding with the expected fraction of expedited repairs constraint (9) for repair resource $r \in R$ and let $v_{m}$ denote the dual variable of $(R M P)$ corresponding to constraint (10) that assures that for each SKU $m \in M$ a convex combination of policies is chosen. (The dual variables $p_{c}, \rho_{r}$, and $v_{m}$ can also be interpreted as Lagrange multipliers of relaxing the corresponding constraints; see Brooks and Geoffrion (1966) and Lübbecke and Desrosiers (2005).) Then, the column generation sub-problem for SKU $m \in M_{r}^{R} \cap M_{c}^{C}$ of $(R M P)$ is given by:

$$
\begin{aligned}
& (S U B(m)) \min _{\left\{\left(\mathbf{S}_{m}, T_{m}\right)\right\}} \sum_{n \in N} c_{a}^{m} S_{m, n}-p_{c} \sum_{n \in N_{l}} E B O_{m, n}\left(\mathbf{S}_{m}, T_{m}\right)-\rho_{r} \delta_{m}^{r} E X P_{m}\left(T_{m}\right)-v_{m} \\
& \text { subject to } \quad \mathbf{S}_{m} \in \mathbb{N}_{0}^{|N|}, \quad T_{m} \in \mathbb{N}_{0} .
\end{aligned}
$$

If a feasible solution to $(S U B(m))$ exists with a negative objective value, then the objective of $(R M P)$ can be improved by adding this policy to $K_{m}^{r e s}$ and solving $(R M P)$ with the larger set $K_{m}^{r e s}$. An optimal solution for $(R M P)$ is also an optimal solution for $(M P)$ if for none of the SKUs a policy with negative reduced costs exists.

In the next section, we present an exact solution method to solve $(S U B(m))$. However, we remark that all policies that yield a negative objective value for $(S U B(m))$, can improve 
the solution of $(R M P)$. Hence, we do not necessarily have to solve $(S U B(m))$ to optimality each time we obtain new dual variables from $(R M P)$.

\subsection{Solving the sub-problem}

This section treats an exact solution method for $(S U B(m))$. All proofs are in Online Appendix A. If we fix the control policy parameters at the central warehouse, then this warehouse simply becomes a supplier with a known stochastic lead time from the perspective of each local warehouse. Hence, for fixed $T_{m}$ and $S_{m, 0}$, each local warehouse $n \in N_{l}$ operates as an independent Newsvendor subsystem, and we can optimize them separately:

TheOrem 1. The optimal $S_{m, n}, n \in N_{l}$, for fixed values of $S_{m, 0}$ and $T_{m}, S_{m, n}^{*}\left(S_{m, 0}, T_{m}\right)$, is the smallest $S_{m, n}\left(S_{m, 0}, T_{m}\right)$ that satisfies

$$
\mathbb{P}\left\{X_{m, n}\left(S_{m, 0}, T_{m}\right) \leq S_{m, n}\left(S_{m, 0}, T_{m}\right)\right\} \geq \frac{p_{c}+c_{a}^{m}}{p_{c}} .
$$

The remaining problem of finding the optimal control policy parameters at the central warehouse is more involved. In fact, it is known that objective function (12) is not convex in $S_{m, 0}$ for a fixed $T_{m}$ and corresponding $S_{m, n}^{*}, n \in N_{l}$ (e.g., Gallego et al. 2007, Rong et al. 2017). Similarly, it can readily be verified that the objective function (12) is also not convex in $T_{m}$ for a fixed $S_{m, 0}$ and corresponding $S_{m, n}^{*}, n \in N_{l}$. Finding the optimal control policy parameters at the central warehouse therefore requires an enumerative search.

To simplify this search, we establish an upper bound on the optimal base stock level at the central warehouse for a given expedite threshold. If the expedite threshold is fixed and the local warehouses carry no inventories, then only the base stock level at the central warehouse can influence the expected backorders at all local warehouses. Hence, the following lemma shows that for fixed $T_{m}$ and $S_{m, n}=0 \forall n \in N_{l}$, the central warehouse also operates as an independent Newsvendor subsystem:

LEMma 1. The optimal $S_{m, 0}$ for fixed $T_{m}$ and $S_{m, n}=0$ for all $n \in N_{l}$, say $\bar{S}_{m, 0}\left(T_{m}\right)$, is the smallest $S_{m, 0}$ that satisfies

$$
\mathbb{P}\left\{X_{m, 0}\left(T_{m}\right) \leq S_{m, 0}\right\} \geq \frac{p_{c}+c_{a}^{m}}{p_{c}}
$$

Observe that if the local warehouses increase their base stock levels, then the amount of inventory that the central warehouse should carry can only decrease (assuming that the expedite threshold is fixed). It is therefore clear that $\bar{S}_{m, 0}\left(T_{m}\right)$ obtained using Lemma 
1 is in fact an upper bound on $S_{m, 0}^{*}\left(T_{m}\right)$ because it assumes no inventories at the local warehouses. This is formalized in the next two results.

LEMma 2. Let $S_{m, n}^{*}\left(S_{m, 0}, T_{m}\right)$ be the optimal value of $S_{m, n}, n \in N_{l}$, for given values of $T_{m}$ and $S_{m, 0}$. Then $S_{m, n}^{*}\left(S_{m, 0}, T_{m}\right)$ is non-increasing in $S_{m, 0}$.

THEOREM 2. $\bar{S}_{m, 0}\left(T_{m}\right)$, as specified in Lemma 1, is an upper bound for $S_{m, 0}^{*}\left(T_{m}\right)$.

Based on the results presented above, we propose the following exact solution method to solve $(S U B(m))$. We set $T_{m}$ to 0 and then search over $T_{m}$. For each value of $T_{m}$, we vary $S_{m, 0}$ over $0 \leq S_{m, 0} \leq \bar{S}_{m, 0}\left(T_{m}\right)$, where $\bar{S}_{m, 0}\left(T_{m}\right)$ is determined using Lemma 1 . For each pair $\left(S_{m, 0}, T_{m}\right)$, we optimize $S_{m, n}$ for all $n \in N_{l}$ using Theorem 1. Since the objective function of $(S U B(m))$ for fixed values of $S_{m, 0}$ and corresponding $S_{m, n}^{*}\left(S_{m, 0}, T_{m}\right), n \in N_{l}$, is not convex in $T_{m}$, we continue the search over $T_{m}$ by examining a few values beyond the last observed local minimum.

\subsection{Constructing a good feasible solution}

When no more policies can be added to $K_{m}^{r e s}$, then a solution to the final version of problem $(R M P)$ provides a lower bound, $C_{P}^{L B}$, on the optimal cost for problem $(P), C_{P}$. In case there are no fractional solutions for any $x_{m}^{k}, m \in M, k \in K_{m}$, this also is an upper bound, $C_{P}^{U B}$, for $C_{P}$. If there are fractional solutions for any $x_{m}^{k}$, we solve the final version of problem $(R M P)$ as an integer linear program. Alvarez et al. $(2013,2015)$ show that this approach yields very good results compared to other methods such as local search algorithms. To speed up the solution process of solving the final version of problem $(R M P)$ as an integer linear program, we use the feasibility pump heuristic of Fischetti et al. (2005), and we stop the solution of the integer linear program as soon as a feasible solution with optimality gap of less than 0.5 percent is found or 1 minute has elapsed (whichever occurs first). This results in a good feasible solution to problem $(P)$. The corresponding cost of this solution is also an upper bound, $C_{P}^{U B}$, for $C_{P}$.

Pseudo-code of the DCG algorithm as well as the greedy heuristic described in the next section can be found in Online Appendix B.

\subsection{A two-step greedy approach}

We now describe a greedy heuristic for problem $(P)$. This greedy heuristic consists of two steps that are executed consecutively. In the first step, we determine, independent of base 
stock level matrix $\mathbf{S}$, expedite threshold vector $T$. Subsequently, based on the vector of expedite thresholds $T$ determined in the first step, we find base stock levels matrix $\mathbf{S}$.

Expediting the repair of a given SKU $m \in M$ implies that fewer parts of $m$ are needed to provide the same availability as when no repairs are expedited. Hence, given that repair resources are limited, we want to expedite the repair of expensive parts more often than cheaper parts. In addition, the cost benefit of expediting the repair of a given SKU $m \in M$ increases in its additional regular lead time, i.e. $t_{m, 0}^{1}$. Hence, given that repair resources are limited, we want to expedite the repair of parts with a greater additional regular repair lead time more often than parts with a smaller additional regular repair lead time.

If there were no restrictions on the aggregate mean fractions of failed parts that are expedited, then, irrespective of base stock levels matrix $\mathbf{S}$, the zero vector would be the optimal vector of expedite thresholds. Hence, in the first step of the greedy heuristic, we set all expedite thresholds $T_{m}, m \in M$, to zero and then start with greedy steps, in which we increase $T_{m}$ leading to the largest decrease in distance to the set of feasible expedite vectors per acquisition cost and additional regular repair lead time.

The first step of the greedy heuristic is formally described as follows. We first partition the set of all expedite thresholds vectors $T$ into a subset $T^{\text {feas }}$ of feasible expedite thresholds vectors, i.e. that respect constraint (5) of problem $(P)$, and a subset $\mathbb{N}_{0}^{|M|} \backslash T^{\text {feas }}$ of infeasible expedite thresholds vectors. Next, for each expedite thresholds vector, we define the distance $d(T)$ to $T^{\text {feas }}$ as

$$
d(T)=\sum_{r \in R}\left(E X P_{r}(T)-\mathcal{E}_{r}^{\max }\right)^{+}
$$

In each greedy step, we have a current solution $T \in \mathbb{N}_{0}^{|M|} \backslash T^{\text {feas }}$, and we look at the ratio of the decrease in distance to $T^{f e a s}$ if $T_{m}, m \in M$, is increased by one unit and the product of the acquisition cost and the additional regular repair lead time. To this end, let $-\Delta_{m} d(T)$ denote the decrease in distance to the set of feasible vectors of expedite thresholds. For a given SKU $m \in M$ that uses repair resource $r \in R$ in the repair of its failed parts, we obtain

$$
\Delta_{m} d(T)=d\left(T+\mathbf{e}_{m}\right)-d(T)=\left(E X P_{r}\left(T+\mathbf{e}_{m}\right)-\mathcal{E}_{r}^{\max }\right)^{+}-\left(E X P_{r}(T)-\mathcal{E}_{r}^{\max }\right)^{+}
$$

where $\mathbf{e}_{m}$ is an $|M|$-dimensional vector with a 1 on position $m$ and zero otherwise. 
Since the Erlang loss formula, and thus $\operatorname{EXP}\left(T_{m}\right)$, is convex and decreasing in $T_{m}$ (e.g., Messerli 1972), it follows that $-\Delta_{m} d(T) \geq 0$ for all $m \in M$. The ratio $\Gamma_{m}^{T}=\frac{-\Delta_{m} d(T)}{t_{m, 0}^{1} c_{a}^{m}}$ denotes the decrease in distance to the set of feasible vectors of expedite thresholds per both the acquisition cost and the additional regular repair lead time. During each greedy step, we increase the expedite threshold of SKU $m$ with the highest $\Gamma_{m}^{T}$ to $T_{m}+1$. We continue with these steps until we arrive at a feasible solution $T$ and we denote this solution by $\bar{T}$.

We now proceed with the second step of the greedy heuristic. If there were no restrictions on the aggregate mean numbers of backorders, then, irrespective of the vector of expedite thresholds, the zero matrix would be the optimal base stock levels matrix. Hence, in the second step of the greedy heuristic, we set all base stock levels $S_{m, n}, m \in M, n \in N$, to zero and then start with greedy steps, in which we increase $S_{m, n}$ leading to the largest decrease in distance to the set of feasible base stock levels matrices per acquisition cost.

The second step of the greedy heuristic is formally described as follows. We first partition the set of all base stock levels matrices $\mathscr{S}$ into a subset $\mathscr{S}^{\text {feas }}$ of feasible base stock levels matrices, i.e. that respect constraint (4) of problem $(P)$, and a subset $\mathscr{S} \backslash \mathscr{S}^{\text {feas }}$ of infeasible base stock levels matrices. Next, for each base stock levels matrix, we define the distance $d(\mathbf{S}, \bar{T})$ to $\mathscr{S}^{\text {feas }}$ as

$$
d(\mathbf{S}, \bar{T})=\sum_{c \in C}\left(E B O_{c}(\mathbf{S}, \bar{T})-\mathcal{B}_{c}^{\max }\right)^{+}
$$

In each greedy step, we have a current solution $\mathbf{S} \in \mathscr{S} \backslash \mathscr{S}^{\text {feas }}$, and we look at the ratio of the decrease in distance to $\mathscr{S}^{\text {feas }}$ and the acquisition cost if $S_{m, n}, m \in M, n \in N$, is increased by one unit. To this end, let $-\Delta_{m, n} d(\mathbf{S}, \bar{T})$ denote the decrease in distance to the set of feasible base stock levels matrices. For each SKU $m \in M$ and warehouse $n \in N$, let $\mathbf{E}_{m, n}$ be an $|M| \times|N|$ matrix with positions $\left(m^{\prime}, n^{\prime}\right), m^{\prime} \in M, n^{\prime} \in N$, with ones on positions $m$ and $n$ and zero otherwise. Then, for a given SKU $m \in M$ of capital good type $c \in C$, we obtain

$$
\begin{aligned}
\Delta_{m, n} d(\mathbf{S}, \bar{T}) & =d\left(\mathbf{S}+\mathbf{E}_{m, n}, \bar{T}\right)-d(\mathbf{S}, \bar{T}) \\
& =\left(E B O_{c}\left(\mathbf{S}+\mathbf{E}_{m, n}, \bar{T}\right)-\mathcal{B}_{c}^{\max }\right)^{+}-\left(E B O_{c}(\mathbf{S}, \bar{T})-\mathcal{B}_{c}^{\text {max }}\right)^{+}
\end{aligned}
$$

Increasing the base stock level of a given SKU $m \in M$ at the central warehouse has a decreasing effect on the expected backorders at all local warehouses $n \in N_{l}$, and no 
effect on the expected backorders of all other SKUs. Moreover, increasing the base stock level of a given SKU $m \in M$ at some local warehouse $n \in N_{l}$ has a decreasing effect on the expected backorders for that SKU at that local warehouse and no effect on all other expected backorders. These assertions are easily verified along similar lines as the proof of Lemma 2. It then immediately follows that $-\Delta_{m, n} d(\mathbf{S}, \bar{T}) \geq 0$ for all $m \in M$ and $n \in N$.

The ratio $\Gamma_{m, n}^{\mathbf{S}}=\frac{-\Delta_{m, n} d(\mathbf{S}, \bar{T})}{c_{a}^{m}}$ denotes the decrease in distance to the set of feasible base stock levels matrices per acquisition cost. During each greedy step, we increase the base stock level of SKU $m$ at warehouse $n$ with the highest $\Gamma_{m, n}^{\mathrm{S}}$ to $S_{m, n}+1$. We continue with these steps until we arrive at a feasible solution $\mathbf{S}$.

\section{Expediting repairs at additional costs}

We have considered a constraint on the aggregate mean fraction of failed parts that are expedited per repair resource so far. This constraint models the agreements between repair shop managers and inventory managers that determine how much of the total stream of failed parts can be expedited per repair resource. When there is an internal repair shop, these agreements may for example relate to the available expedited repair capacity per repair resource. There might be settings where it is relatively easy to obtain the exact costs associated with expediting a repair (e.g. in case of an external repair shop). In this section, we show that our DCG algorithm can be applied in this alternate setting almost immediately. In fact, as we will see shortly, there exists an equivalence relation between this setting and our original setting for a certain expediting cost structure.

When expediting has an additional cost, the objective is to minimize a total cost rate per time unit consisting of the total depreciation cost rate in spare parts and the total expediting cost rate, where the depreciation cost rate is obtained by depreciating the total initial investment costs in spare parts over their useful lifespan. We note that an alternative, but mathematically equivalent, formulation is to minimize a total initial cost consisting of both the total investment costs in spare parts (as in the original setting) and the total expected discounted expediting costs over an infinite horizon (see Online Appendix C).

We first introduce some additional notation. Let the cost of expediting the repair of one part of SKU $m \in M$ be denoted by $c_{e}^{m}$. We assume that this cost is linearly proportional to the acquisition cost of that SKU, so that expediting the repair of more expensive SKUs is also more expensive than expediting the repair of cheaper SKUs. That is, $c_{e}^{m}=\kappa \cdot c_{a}^{m}$, 
where $\kappa>0$ denotes what we refer to as the expediting cost multiplier. The depreciation cost rate of each part of SKU $m \in M$ is denoted by $c_{d}^{m}$, and can be obtained by linearly depreciating its acquisition $\operatorname{cost} c_{a}^{m}$ over its useful lifespan $\tau$, i.e. $c_{d}^{m}=c_{a}^{m} / \tau$. For a given control policy $(\mathbf{S}, T)$, the total depreciation cost rate in spare parts is defined as $C_{d}(\mathbf{S})=\sum_{m \in M} \sum_{n \in N} c_{d}^{m} S_{m, n}$ and the total repair expediting cost rate as $C_{e}(T)=$ $\sum_{m \in M} c_{e}^{m} \lambda_{m, 0} E X P_{m}\left(T_{m}\right)$. The mathematical formulation of the alternate decision problem, which we call problem $(\widehat{P})$, is given as follows:

$$
\begin{array}{lll}
(\widehat{P}) \quad \min _{\{\mathbf{S}, T\}} & C_{d}(\mathbf{S})+C_{e}(T) \\
\text { subject to } & E B O_{c}(\mathbf{S}, T) \leq \mathcal{B}_{c}^{\text {max }}, \quad \forall c \in C \\
& \mathbf{S} \in \mathscr{S}, \quad T \in \mathbb{N}_{0}^{|M|} .
\end{array}
$$

We note that problem $(\widehat{P})$ constitutes the Lagrangian relaxation of our original decision problem $(P)$ when either (i) each SKU has a dedicated repair resource or more generally when (ii) expediting costs are identical for all SKUs that use the same repair resource. The cost of expediting the repair of SKU $m\left(c_{e}^{m}\right)$ is then equivalent to the Lagrange multiplier $\left(\rho_{r}\right)$ for the resource needed for the repair of SKU $m$ divided by the constant $\lambda_{m, 0}$.

We now proceed to show how the DCG algorithm can be altered so that it applies to problem $(\widehat{P})$. We note that the main structure of the DCG algorithm remains unchanged; only its master problem and column generation sub-problem should be modified. The master problem of the DCG algorithm is now given by problem $(\widehat{M P})$ :

$$
\begin{array}{cl}
(\widehat{M P})_{\left\{x_{m}^{k}: m \in M, k \in K_{m}\right\}} \sum_{m \in M} \sum_{n \in N} \sum_{k \in K_{m}} c_{d}^{m} S_{m, n}^{k} x_{m}^{k}+\sum_{m \in M} \sum_{k \in K_{m}} c_{e}^{m} \lambda_{m, 0} \operatorname{EXP}_{m}\left(T_{m}^{k}\right) x_{m}^{k} \\
\text { subject to } \sum_{m \in M_{c}^{C}} \sum_{n \in N_{l}} \sum_{k \in K_{m}} E B O_{m, n}\left(\mathbf{S}_{m}^{k}, T_{m}^{k}\right) x_{m}^{k} \leq \mathcal{B}_{c}^{m a x}, & \forall c \in C \\
\sum_{k \in K_{m}} x_{m}^{k}=1, & \forall m \in M \\
x_{m}^{k} \geq 0, & \forall m \in M, \forall k \in K_{m} .
\end{array}
$$

The corresponding column generation sub-problem for SKU $m \in M_{c}^{C}$ is now given by:

$$
\begin{aligned}
& (\widehat{S U B}(m)) \min _{\left\{\left(\mathbf{S}_{m}, T_{m}\right)\right\}} \sum_{n \in N} c_{d}^{m} S_{m, n}+c_{e}^{m} \lambda_{m, 0} E X P_{m}\left(T_{m}\right)-p_{c} \sum_{n \in N_{l}} E B O_{m, n}\left(\mathbf{S}_{m}, T_{m}\right)-v_{m} \\
& \text { subject to } \quad \mathbf{S}_{m} \in \mathbb{N}_{0}^{|N|}, \quad T_{m} \in \mathbb{N}_{0} .
\end{aligned}
$$


For a fixed expedite threshold $T_{m},(\widehat{S U B}(m))$ has exactly the same structure as the column generation sub-problem for our original setting. Hence, all results on $(S U B(m))$ presented in Section 5.2 remain to hold for $(\widehat{S U B}(m))$ exactly as stated. In particular the solution method described at the end of that section immediately applies to $(\widehat{S U B}(m))$.

\section{Computational study}

The computational study in this section consists of two parts. In Section 7.1, we report on a case study at NS and present managerial insights. In Section 7.2 and Online Appendix E, we evaluate and benchmark the performance of our solution approaches in an extensive numerical study based on a large test bed of randomly generated instances. We do so both for constrained expediting, i.e. problem $(P)$, and expediting at additional costs, i.e. problem $(\widehat{P})$. We programmed our solution approaches as single threaded applications in $\mathrm{C}$ with GLPK as the solver of both linear and integer linear programs. All computations were carried out on a Windows PC (32 bit) with an Intel Quad Core 2.20 GHz processor and 8 GB RAM.

\subsection{Case study at NS}

NS is the principal passenger railway operator in the Netherlands. Its fleet consists of 900 rolling stock units, divided over twelve different train series. The spare parts inventory system of NS consists of one central warehouse and twelve local warehouses. There is a large repair center incident to the central warehouse. This repair center consists of multiple repair shops, each responsible for a different repair resource.

7.1.1. Setup and objective This case study is focused on the VIRM train series; our case study therefore involves one capital good type, i.e. $|C|=1$. The VIRM series consist of 176 rolling stock units, all of which are being operated as intercity trains that connect most cities in the Netherlands. We consider the six most important warehouses where the VIRM train series is maintained and leave a handful of locations with only incidental demand out of scope; hence, $\left|N_{l}\right|=6$.

We select 74 critical SKUs that occur in the configuration of the VIRM series. Of these SKUs, 30 require a mechanical resource for their repair and 44 require an electronic resource for their repair; hence, $\left|M_{r}^{R}\right|=2$. The regular and expedited repair lead time for both repair resources is three weeks and one week, respectively. The transportation time is one week and includes administration time and shipment time from the central warehouses to 
all local warehouses. The acquisition costs of all SKUs range between 150.52 and 23,399.64 euros, and are 2,282.54 euros on average. We applied Maximum Likelihood Estimation to 5 years of historical failure data to estimate the demand intensities for each SKU. This estimation procedure leads to demand intensities that vary between 1 and 174 per year. In Figure 4, we plot and classify each SKU based on its normalized demand intensity and normalized acquisition cost. This classification will be important when we discuss the results of our case study.

\section{Figure 4 Scatterplot of SKUs in case study}

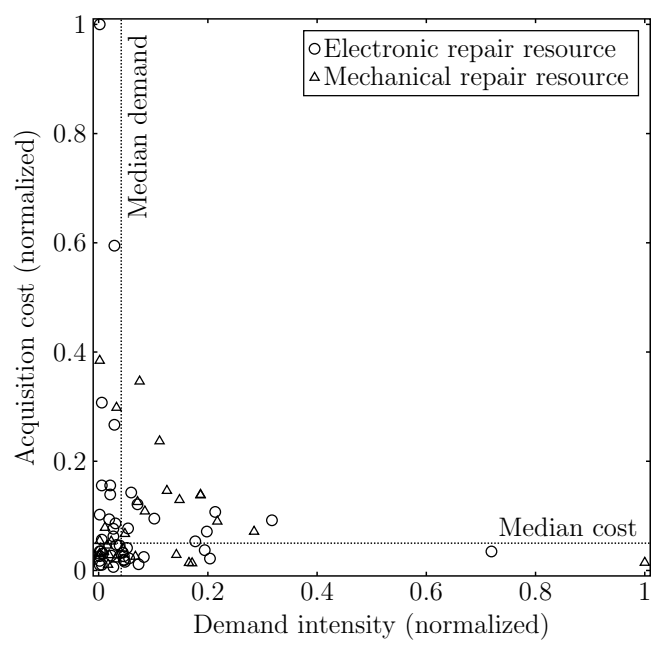

The current practice at NS can be described as follows. On a strategic level, inventory managers decide upon stocking levels using a single-item single-echelon model of the commercial package Servigistics (formerly Xelus Parts Planning). This model does not take into account that NS has the possibility to expedite the repair of parts in short supply. Expediting decisions are then made by inventory managers and repair shop managers together operationally on a weekly basis. For the electronic and mechanical repair shop, we observe from historical data that 30 percent of the total stream of failed parts is expedited.

The main objective of this case study is twofold. First, we want to determine the reductions in investment costs that can be achieved when our solution approaches are used to achieve the same performance as the current approach of NS achieves. Second, and more importantly, we want to understand how a dynamic repair policy can be leveraged to reduce the total investment costs in spare parts while meeting availability targets.

Our benchmark for the case study is the current solution that NS uses. In this solution, the investment in each spare part is determined by the stocking model of Servigistics. For 
this investment decision, we determine the best achievable availability performance by optimizing expediting decisions and stock placement within our modeling framework. We then use the DCG algorithm and the greedy heuristic to find alternative investment decisions with at least the same availability performance. Further details regarding this benchmark, including the single-item model of Servigistics, are provided in Online Appendix F.

7.1.2. Results and managerial implications Table 2 shows the normalized investment costs of the three approaches to make investment decisions and the corresponding availability performance and expediting fractions. We observe that the greedy heuristic leads to an investment costs reduction of $52.43 \%$ compared to the current approach. As expected, the DCG algorithm has an even higher cost benefit with 53.55\%. We remark that these savings are in the same order of magnitude as in other real-life applications that have compared a system approach with a single-item approach in a multi-item spare parts context. See, for example, Sherbrooke (2004) and Van Houtum and Kranenburg (2015) for various applications at the US Air Force and the Royal Dutch Navy, respectively. (We see later in Section 7.2.2 that our solution approaches also lead to high savings in the setting of NS when we benchmark against a state-of-the-art multi-item model).

Apart from the substantial investment costs reductions that can be reaped, it is interesting to note that the gap between the DCG algorithm and the greedy heuristic is small. Later when we discuss the results of our numerical experiments in Section 7.2, we will see that this holds across a large variety of industrial size problem instances.

Table 2 Main results case study at NS

\begin{tabular}{lcccc}
\hline Solution approach & Investment costs (normalized) & $E B O_{\text {VIRM }}$ & $E X P_{\text {electronic }}(\%)$ & $E X P_{\text {mechanical }}(\%)$ \\
\hline Current approach NS & 100 & 13.70 & 29.82 & 29.98 \\
Greedy heuristic & 47.57 & 13.65 & 29.99 & 29.97 \\
DCG algorithm & 46.45 & 13.65 & 29.99 & 29.94 \\
\hline
\end{tabular}

Recall that we classified all SKUs into four distinct SKU groups based on their acquisition costs and demand intensities. To illustrate how the decisions of our new approaches realize the substantial cost reductions reported in Table 2, we will investigate the performance of each of these SKU groups. To facilitate presentation, we first introduce some additional notation. Let $G$ denote the set of different SKU groups, hence $G=$ $\{$ high demand, low demand $\} \times\{$ high cost, low cost $\}$ and thus $|G|=4$. The SKUs that 
belong to group $g \in G$ are contained in the set $M_{g}^{G}$. For each of the three investment decisions consisting of acquired stock $\mathbf{S}$ and expedite thresholds $T$, we now calculate the following performance measures:

$$
\begin{aligned}
\overline{\operatorname{EXP}}_{g}(T) & =\left(\sum_{m \in M_{g}^{G}} \operatorname{EXP}_{m}\left(T_{m}\right)\right) /\left|M_{g}^{G}\right|, \\
\operatorname{EXP}_{g}(T) & =\sum_{r \in R} \sum_{m \in M_{g}^{G}} \delta_{m}^{r} \operatorname{EXP}_{m}\left(T_{m}\right), \\
\operatorname{STOCK}_{g}(\mathbf{S}) & =\left(\sum_{m \in M_{g}^{G}} \sum_{n \in N} S_{m, n}\right) /\left(\sum_{m \in M_{g}^{G}} \lambda_{m, 0}\right), \\
\operatorname{EBO}_{g}(\mathbf{S}, T) & =\sum_{m \in M_{g}^{G}} \sum_{n \in N_{l}} E B O_{m, n}\left(\mathbf{S}_{m}, T_{m}\right), \text { and } \\
\operatorname{COST}_{g}(\mathbf{S}) & =100 \cdot\left(\sum_{m \in M_{g}^{G}} \sum_{n \in N} c_{a}^{m} S_{m, n}\right) / C(\mathbf{S}),
\end{aligned}
$$

which all provide meaningful information about an SKU group $g \in G$. The mean expedited repair utilization and the total aggregate mean expedited repair utilization of $g$ are given by $\overline{\operatorname{EXP}}_{g}(T)$ and $\operatorname{EXP}(T)$, respectively. Note that $\overline{\operatorname{EXP}}_{g}(T) \in[0,1]$ and $\operatorname{EXP}(T) \in[0,0.6]$. $S T O C K_{g}(\mathbf{S})$ provides a normalized measure of how much stock of all SKUs in $g$ is acquired. The total mean number of backorders for $g$ is given by $E B O_{g}(\mathbf{S}, T)$. Finally, $C O S T_{g}(\mathbf{S})$ measures the relative difference between the investment costs in $g$ and the overall total costs under the investment decision.

Table 3 provides the performance measures for each SKU group $g \in G$ under each of the three investment decisions. For now, we only consider the performance measures of our solution approaches, and we turn our attention to the left upper quadrant: SKUs with low demand intensities and high acquisition costs. As the table indicates, the unavailability due to this group of SKUs is kept relatively low by providing full repair priority to failed parts rather than by investing in spare parts. Similar to Sherbrooke (2004, p.12) in an application at the US Air Force, we observe that for "high cost items [...] the model has allocated a zero or low stock level". Although failed parts always receive expedited repair, this SKU group utilizes only $9.22 \%$ of the total available expediting capacity. Conversely, if we look at the right lower quadrant, SKUs with low acquisition costs and high demand intensities receive almost no expedited repair. Instead, the unavailability due to this group of SKUs is kept relatively low by acquiring large amounts of spare parts.

For the other two SKU groups, our solution approaches neither solely invest in spare parts nor solely expedite the repair of failed parts. If we look at the SKUs with low demand intensities and low acquisition costs, we indeed observe that this group has a large amount 
Table 3 Performance measures per SKU group under each solution approach

\begin{tabular}{|c|c|c|c|c|c|c|c|c|}
\hline & \multicolumn{6}{|c|}{ Solution approach } & \multirow[b]{2}{*}{ Measure } \\
\hline & & DCG & Greedy & Current & DCG & Greedy & Current & \\
\hline \multirow{10}{*}{ 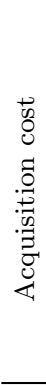 } & \multirow{5}{*}{ 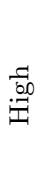 } & 100 & 100 & 9.34 & 67.08 & 65.56 & 34.63 & $\overline{E X P}(\%)$ \\
\hline & & 9.22 & 9.22 & 1.25 & 46.73 & 41.96 & 27.25 & $\operatorname{EXP}(\%)$ \\
\hline & & 0.00 & 0.00 & 92.57 & 15.62 & 16.17 & 23.98 & STOCK \\
\hline & & 0.00 & 0.00 & 37.75 & 52.51 & 54.37 & 46.18 & $C O S T$ \\
\hline & & 1.94 & 1.94 & 0.92 & 8.39 & 8.54 & 5.76 & $E B O$ \\
\hline & \multirow{5}{*}{ בְּ } & 55.57 & 61.39 & 13.43 & 5.11 & 10.83 & 36.60 & $\overline{E X P}(\%)$ \\
\hline & & 2.04 & 4.18 & 1.26 & 1.94 & 4.60 & 30.04 & $\operatorname{EXP}(\%)$ \\
\hline & & 66.00 & 66.00 & 104.5 & 44.24 & 44.24 & 23.78 & $S T O C K$ \\
\hline & & 5.08 & 4.22 & 4.92 & 42.41 & 41.41 & 11.14 & $C O S T$ \\
\hline & & 1.09 & 1.05 & 0.96 & 2.22 & 2.12 & 6.06 & $E B O$ \\
\hline & & & Low & & & High & & \\
\hline & & & & Deman & ntensi & & & \\
\hline
\end{tabular}

of normalized acquired stock as well as a high average expedited repair utilization. As a result, this group has the smallest mean number of backorders of all groups. The impact on the total investment costs and the total available expediting capacity is however small as both demand intensities and acquisition costs are low.

From the right upper quadrant, we observe that a large part of the available expediting capacity is utilized by the group of SKUs with both high demand intensities and high acquisition costs. Although the investment costs in this group are more than half of the total costs of the investment decision, the normalized acquired stock is relatively small. Finally, with more than 8 expected backorders, the unavailability due to this group of SKUs is significantly larger than all other groups.

Our integrated solution approaches thus lead to well-balanced investment decisions in which we acquire large amounts of spare parts of SKUs with low acquisition costs. In doing so, we maximize the availability of these SKUs at relatively low investment costs. Almost all available expediting capacity is then leveraged to dynamically prioritize the repair of failed parts with high acquisition costs, which allows us to refrain from excessively acquiring spare parts with such high costs. The current approach leads to a less balanced investment decision. As Table 3 indicates, the current approach invests heavily in spare parts with high acquisition costs and mainly prioritizes the repair of failed parts of SKUs with high demand intensities.

We emphasize that the dynamic repair policy requires real-time information about the repair pipeline in deciding upon expedited repairs. This should be taken into account when 
implementing our solution approaches in practice. In case of NS, this real-time information is obtained through modern tracking technology that utilizes radio-frequency identification.

\subsection{Numerical experiments}

In the previous section, we have described how our solution approaches leverage a dynamic repair policy to reduce the total investment costs in spare parts while satisfying availability and expediting constraints. In this section and Online Appendix E, we assess the value of having such an advanced dynamic repair policy in the first place. We also investigate whether our solution approaches find solutions that are close to optimal and whether they find such solutions within reasonable time. We answer these questions for both the original decision problem that was also faced by NS, i.e. problem $(P)$, and the alternate decision problem in which expediting a repair comes at an additional cost, i.e. problem $(\widehat{P})$.

7.2.1. Test beds Our test bed for decision problem $(P)$ consists of 2592 randomly generated problem instances obtained by permuting all input parameters over multiple levels that are based on representative data for the capital goods industry. This test bed consists of both symmetric instances, in which the demand intensities across all local warehouses are identical but varied for different SKUs, and asymmetric instances, in which the demand intensities are varied across all local warehouses and SKUs. Our test bed for

decision problem $(\widehat{P})$ also consists of 2592 instances, and is identical to the test bed for decision problem $(P)$ except for the two input parameters that are specific to problem $(P)$, i.e. $\mathcal{E}_{r}^{\max }$ and $|R|$. We replace those two input parameters with the two input parameters that are specific to problem $(\widehat{P})$, i.e. the expediting cost multiplier $\kappa$ and the useful life span $\tau$. Further details regarding both test beds are relegated to Online Appendix E.

7.2.2. Results when expediting is constrained We first consider decision problem $(P)$. To evaluate the effectiveness of our solution approaches in solving this problem, we compute a feasible solution for each generated instance using both solution approaches and we measure the relative difference between the total cost obtained by the solution approach and the corresponding lower bound. That is, $\% G A P=100 \cdot\left(C_{P}^{U B}-C_{P}^{L B}\right) / C_{P}^{L B}$, where $C_{P}^{L B}$ is obtained using the method described in Section 5.1 and where $C_{P}^{U B}$ is obtained using the method described in Section 5.3 in case of the DCG algorithm, or using the method described in Section 5.4 in case of the greedy heuristic. 
To quantify the value of our dynamic repair policy, we create a state-of-the-art benchmark instance for each original instance of problem $(P)$ that we generate. This benchmark instance is identical to the original instance except that it is not possible to differentiate repair lead times through expediting. The mean repair lead time of this benchmark instance is then kept below the mean repair lead time of the original instance. This is achieved as follows: We set $\mathcal{E}_{r}^{\max }$ to 1.0 for each repair resource $r \in R$ in the original instance such that it is feasible (and optimal) to expedite all repairs. We then change the expedited lead time $t_{m, 0}^{2}$ of each SKU $m \in M$ to the shortest mean repair lead time possible in the feasible solution to the original instance. For a given SKU $m \in M$ that requires resource $r \in R$ for its repair, this shortest mean repair lead time is $\left(1-\mathcal{E}_{r}^{\max }\right) \cdot\left(t_{m, 0}^{1}+t_{m, 0}^{2}\right)+\mathcal{E}_{r}^{\max } \cdot t_{m, 0}^{2}$. For this benchmark instance, we compute a lower bound on the optimal cost using the method described in Section 5.1. We denote this lower bound by $C_{B M}^{L B}$ and we compare it with $C_{P}^{U B}$ of the original instance, obtained by the DCG algorithm. That is, $\% R E D=100 \cdot\left(C_{B M}^{L B}-C_{P}^{U B}\right) / C_{B M}^{L B}$, where $\% R E D$ will indicate how much stock investment reductions can be achieved because of the possibility to expedite the repair of parts in short supply.

Aggregated results of the numerical experiments involving problem $(P)$ are presented in Table 4. Detailed results are provided in Online Appendix E. We note that the solutions to the problem instances generally exhibit the same behavior as extensively described in the case study.

Table 4 Aggregated results numerical experiments involving problem $(P)$

\begin{tabular}{|c|c|c|c|c|c|c|c|c|c|c|}
\hline \multirow[b]{3}{*}{ Problem instances } & \multicolumn{4}{|c|}{ DCG algorithm } & \multicolumn{4}{|c|}{ Greedy heuristic } & \multirow{2}{*}{\multicolumn{2}{|c|}{$\begin{array}{c}\text { Benchmark } \\
\% R E D\end{array}$}} \\
\hline & \multicolumn{2}{|c|}{$\% G A P$} & \multicolumn{2}{|c|}{ CPU time $(s)$} & \multicolumn{2}{|c|}{$\% G A P$} & \multicolumn{2}{|c|}{ CPU time $(s)$} & & \\
\hline & Avg & $\operatorname{Max}$ & Avg & Max & Avg & Max & Avg & Max & Avg & Max \\
\hline Asymmetric & 0.26 & 0.75 & 90.08 & 939.03 & 1.07 & 3.15 & 1.55 & 11.47 & 7.95 & 18.81 \\
\hline Symmetric & 0.28 & 0.77 & 111.34 & 1271.85 & 3.69 & 8.43 & 1.75 & 12.85 & 7.92 & 19.61 \\
\hline Total & 0.27 & 0.77 & 100.71 & 1271.85 & 2.38 & 8.43 & 1.66 & 12.85 & 7.94 & 19.61 \\
\hline
\end{tabular}

The numerical experiments indicate that both solution approaches perform very well. The average optimality gaps of the DCG algorithm over the asymmetric and the symmetric problem instances are only 0.26 and 0.28 , respectively. The optimality gaps of the greedy heuristic are slightly larger with 1.07 and 3.69 over the asymmetric and the symmetric problem instances, respectively. The greedy heuristic is the most efficient heuristic in terms 
of computation time. Although the computation time of the DCG algorithm is considerably higher, it is still acceptable given the size and strategic nature of the decision problem.

The stock investment reductions that can be achieved because of the possibility to expedite the repair of parts in short supply are quite high with an average stock investment reduction of around 7.9 percent and even reductions of up to 19.61 percent. Before we continue with analyzing the alternate setting, we briefly return to the case study at NS. The value of a dynamic repair policy in their setting is substantial with a stock investment reduction of 36.40 percent. This is not surprising because our numerical experiments indicate that the value of a dynamic repair policy increases in the additional regular repair lead time or in the fraction of total demand that may be expedited. Both input parameters are slightly larger in the case study than in the problem instances of our test bed.

7.2.3. Results when expediting comes at additional costs We now proceed with decision problem $(\widehat{P})$. The optimality gap of the DCG algorithm in this setting is defined as $\% G A P=100 \cdot\left(C_{\widehat{P}}^{U B}-C_{\widehat{P}}^{L B}\right) / C_{\widehat{P}}^{L B}$, where $C_{\widehat{P}}^{U B}$ and $C_{\widehat{P}}^{L B}$ are defined in a similar way as in the previous section, and can they be obtained using the methods described in Section 6 .

Recall that in this alternate setting, there is an external (or internal) repair shop that charges an additional cost whenever we decide to expedite the repair of a failed part. Even though we thus have to pay a cost premium for a shorter repair lead time, we can leverage this flexibility when inventory is critically low. Our model anticipates precisely these future expediting decisions when deciding upon stocking levels. To quantify the value of our dynamic repair policy in this setting, we create a state-of-the-art benchmark instance that is identical to the original instance except that we do not have the possibility to shorten the repair lead time at the expense of a cost premium when backorders are imminent. Hence, similar to the benchmark for problem $(P)$, we can only decide upon stocking levels in meeting availability constraints. This benchmark is created as follows: In the original instance we set $c_{e}^{m}=\infty$ for each SKU $m \in M$ such that it is optimal to not expedite any repairs. For this benchmark instance, we compute a lower bound on the optimal cost using the method described in Section 6 . We denote this lower bound by $C_{\overrightarrow{B M}}^{L B}$ and we compare it with $C_{\widehat{P}}^{U B}$ of the original instance, obtained by the DCG algorithm. That is, $\% R E D=100 \cdot\left(C_{B M}^{L B}-C_{\vec{P}}^{U B}\right) / C_{B M}^{L B}$, where $\% R E D$ will now indicate by how much the total cost rate can be reduced because of the possibility to dynamically expedite repairs at the expense of a cost premium when inventory is critically low. 
The aggregated results of the numerical experiments involving problem $(\widehat{P})$ are presented in Table 5. Detailed results are again relegated to Online Appendix E. We can draw three

\begin{tabular}{|c|c|c|c|c|c|c|c|c|}
\hline \multirow[t]{4}{*}{ Table 5} & \multicolumn{8}{|c|}{ Aggregated results numerical experiments involving problem $(\widehat{P})$} \\
\hline & \multicolumn{4}{|c|}{ DCG algorithm } & \multirow{2}{*}{\multicolumn{2}{|c|}{$\frac{\text { Benchmark }}{\% R E D}$}} & \multirow{2}{*}{\multicolumn{2}{|c|}{$\begin{array}{c}\text { Expediting } \\
E X P(\%)\end{array}$}} \\
\hline & \multicolumn{2}{|c|}{$\% G A P$} & \multicolumn{2}{|c|}{ CPU time $(s)$} & & & & \\
\hline & Avg & Max & Avg & Max & Avg & Max & Avg & $\operatorname{Max}$ \\
\hline & 0.18 & 0.54 & 176.21 & 2757.51 & 4.86 & 29.29 & 17.37 & 94.02 \\
\hline
\end{tabular}

main conclusions from these results. First, the DCG algorithm performs even better when it is applied to problem $(\widehat{P})$ : The average and maximum $\%$ GAP are only 0.18 and 0.54 percent, respectively. Second, although the computation time of the DCG algorithm is higher for problem $(\widehat{P})$ than for problem $(P)$, it is still well within acceptable bounds given the size and strategic nature of the decision problem. Finally and most importantly, we find that anticipating expediting decisions that will be made later with investment decisions in repairable spare parts leads to substantial savings, even when those expedited repairs come at a cost premium. Indeed, the possibility to expedite the repair of failed parts at additional costs is effective in reducing the total cost rate with average reductions of around 4.9 percent and even reductions up to 29.29 percent.

\section{Concluding remarks}

We have considered a multi-item two-echelon spare parts inventory system, where each warehouse keeps multiple repairable types to maintain several types of capital goods, and where the repair shop at the central warehouse has two options for the repair of each defective part: a regular repair option and an expedited repair option. Irrespective of the repair option, each defective part uses a certain resource for its repair. Assuming a dualindex policy at the central warehouse and base stock control at the local warehouses, we have proposed an exact evaluation procedure for a given control policy.

To find an optimal control policy, we have formulated an optimization problem aimed at minimizing the total investment costs under constraints on both the aggregate mean number of backorders per capital good type and the aggregate mean fraction of repairs that are expedited per repair resource. We have shown how this non-linear non-convex integer programming problem can be decomposed into independent Newsvendor type sub-problems per repairable type, which subsequently allows us to use column generation algorithms. 
As an alternative solution approach, we have presented an efficient greedy heuristic. Both solution approaches perform very well across a large test bed of industrial size.

We have shown that a dynamic repair policy is effective in reducing the stock investment needed to meet availability requirements for multiple types of capital goods while also satisfying expedited repair constraints for multiple repair resources. Our numerical experiments further show that such reductions remain attainable when expediting repairs comes at additional costs rather than being constrained. Based on a case study at NS, we have shown that our solution approaches lead to well-balanced investment decisions in which large amounts of spare parts of SKUs with low acquisition costs are acquired. In doing so, the availability of these SKUs can be maximized at relatively low investment costs. Almost all available expediting capacity can then be leveraged to dynamically prioritize the repair of failed parts with high acquisition costs, which allows us to refrain from excessively acquiring spare parts with such high costs.

The research in this paper can be extended in two important ways. The first class of possible extensions would consider additional transportation modes, either from the central warehouse to the local warehouses, or in between the local warehouses themselves. The former relates to expedited transportation while the latter relates to so-called lateral transhipments. Both can serve as an emergency mode in case a local warehouse is out of stock, or as an expedited transport mode in case of imminent downtime. The second class of possible extensions would allow for demand to be non-stationary. Such processes can capture demand fluctuations for repairable spare parts over time, which might occur in practice due to for instance periodic inspections or revisions of equipment. Similar to Arts et al. (2016), assuming that demand for each repairable type is a Markov modulated Poisson process would then be a promising approach.

\section{Acknowledgments}

The authors thank Collin Drent, Geert-Jan van Houtum, Wouter Fleuren, Bob Huisman, and Benny Mantin for several helpful discussions. The authors also thank the editorial team for their constructive feedback on earlier versions of this work. The first author is grateful to the Maintenance Development department of Dutch Railways for providing a stimulating research environment. He also gratefully acknowledges the support of the National Research Fund of Luxembourg through AFR grant 12451704. The second author acknowledges support from the Netherlands Organisation for Scientific Research through VENI grant 451$16-025$. 


\section{References}

Adan, I.J.B.F., A. Sleptchenko, G.J.J.A.N. Van Houtum. 2009. Reducing costs of spare parts supply systems via static priorities. Asia-Pacific Journal of Operational Research 26(04) 559-585.

Alfredsson, P., J. Verrijdt. 1999. Modeling emergency supply flexibility in a two-echelon inventory system. Management Science 45(10) 1416-1431.

Alvarez, E.M., M.C. Van der Heijden, W.H.M. Zijm. 2013. The selective use of emergency shipments for service-contract differentiation. International Journal of Production Economics 143(2) 518-526.

Alvarez, E.M., M.C. Van der Heijden, W.H.M. Zijm. 2015. Service differentiation in spare parts supply through dedicated stocks. Annals of Operations Research 231(1) 283-303.

Arts, J.J. 2017. A multi-item approach to repairable stocking and expediting in a fluctuating demand environment. European Journal of Operational Research 256(1) 102-115.

Arts, J.J., R.J.I. Basten, G.J.J.A.N. Van Houtum. 2016. Repairable stocking and expediting in a fluctuating demand environment: Optimal policy and heuristics. Operations Research 64(6) 1285-1301.

Arts, J.J., M. Van Vuuren, G.P. Kiesmüller. 2011. Efficient optimization of the dual-index policy using markov chains. IIE Transactions 43(8) 604-620.

Basten, R.J.I., G.J.J.A.N. Van Houtum. 2014. System-oriented inventory models for spare parts. Surveys in Operations Research and management science 19(1) 34-55.

Bitton, S., I. Cohen, M.A. Cohen. 2019. Joint repair sourcing and stocking policies for repairables using erlang-a and erlang-b queueing models. IISE Transactions 51(10) 1151-1166.

Brooks, R., A. Geoffrion. 1966. Finding everett's lagrange multipliers by linear programming. Operations Research 14(6) 1149-1153.

Caggiano, K.E., J.A. Muckstadt, J.A. Rappold. 2006. Integrated real-time capacity and inventory allocation for reparable service parts in a two-echelon supply system. Manufacturing $\mathscr{E}$ Service Operations Management 8(3) 292-319.

Cohen, M., P.V. Kamesam, P. Kleindorfer, H. Lee, A. Tekerian. 1990. Optimizer: Ibm's multi-echelon inventory system for managing service logistics. Interfaces 20(1) 65-82.

Cohen, M.A., Y.S. Zheng, V. Agrawal. 1997. Service parts logistics: a benchmark analysis. IIE transactions 29(8) 627-639.

Dantzig, G.B., P. Wolfe. 1960. Decomposition principle for linear programs. Operations Research 8(1) $101-111$.

Drent, M. 2017. Stocking and expediting in two-echelon spare parts inventory systems under system availability constraints. Master's thesis, Eindhoven University of Technology. URL https://research.tue.nl/ en/studentTheses/stocking-and-expediting-in-two-echelon-spare-parts-inventory-syst.

Feng, Q., S.P. Sethi, H. Yan, H. Zhang. 2006. Are base-stock policies optimal in inventory problems with multiple delivery modes? Operations Research 54(4) 801-807. 
Fischetti, M., F. Glover, A. Lodi. 2005. The feasibility pump. Mathematical Programming 104(1) 91-104.

Gallego, G., Ö. Özer, P. Zipkin. 2007. Bounds, heuristics, and approximations for distribution systems. Operations Research 55(3) 503-517.

Graves, S.C. 1985. A multi-echelon inventory model for a repairable item with one-for-one replenishment. Management Science 31(10) 1247-1256.

Gross, J.F., J.M. Thompson, C.M. Harris. 2008. Fundamentals of Queueing Theory. 4th ed. WileyInterscience, New York, NY, USA.

Howard, C., J. Marklund, T. Tan, I. Rijnen. 2015. Inventory control in a spare parts distribution system with emergency stocks and pipeline information. Manufacturing 8 Service Operations Management $\mathbf{1 7}(12)$ 142-156.

Kellerer, H., U. Pferschy, D. Pisinger. 2004. Knapsack problems.. Springer, Berlin.

Klosterhalfen, S., G. Kiesmller, S. Minner. 2011. A comparison of the constant-order and dual-index policy for dual sourcing. International Journal of Production Economics 133(1) 302 - 311.

Kranenburg, A.A., G.J.J.A.N. Van Houtum. 2007. Effect of commonality on spare parts provisioning costs for capital goods. International Journal of Production Economics 108(1) 221-227.

Kranenburg, A.A., G.J.J.A.N. Van Houtum. 2009. A new partial pooling structure for spare parts networks. European Journal of Operational Research 199(3) 908-921.

Lam, S. 1977. Queuing networks with population size constraints. IBM J. Res. Devel. 21 370-378.

Lee, H.L. 1987. A multi-echelon inventory model for repairable items with emergency lateral transshipments. Management Science 33(10) 1302-1316.

Loeffen, N. 2012. Repair shop and inventory control for spare parts: min-max versus a lead time interface agreement. Master's thesis, Eindhoven University of Technology. URL https://research.tue.nl/ en/studentTheses/repair-shop-and-inventory-control-for-spare-parts.

Lübbecke, M.E., J. Desrosiers. 2005. Selected topics in column generation. Operations Research 53(6) 1007-1023.

Messerli, E.J. 1972. Proof of a convexity property of the erlang b formula. The Bell System Technical Journal 51(4) 951-953.

Moinzadeh, K., P.K. Aggarwal. 1997. An information based multiechelon inventory system with emergency orders. Operations Research 45(5) 694-701.

Moinzadeh, K., C.P. Schmidt. 1991. An (s-1, s) inventory system with emergency orders. Operations Research 39(2) 308-321.

Muckstadt, J.A. 1973. A model for a multi-item, multi-echelon, multi-indenture inventory system. Management Science 20(4) 472-481. 
Muckstadt, J.A. 2005. Analysis and algorithms for service parts supply chains. Springer Science \& Business Media.

Pyke, D.F. 1990. Priority repair and dispatch policies for reparable-item logistics systems. Naval Research Logistics (NRL) 37(1) 1-30.

Rong, Y., Z. Atan, L.V. Snyder. 2017. Heuristics for basestock levels in multiechelon distribution networks. Production and Operations Management 26(9) 1760-1777.

Rustenburg, W.D. 2000. A system approach to budget-constrained spare parts management. Ph.D. thesis, Eindhoven University of Technology.

Rustenburg, W.D., G.J.J.A.N. Van Houtum, W.H.M. Zijm. 2001. Systeemgericht spare parts management bij de nederlandse koninklijke marine. Bedrijfskunde 73(2) 28-39.

Sheopuri, A., G. Janakiraman, S. Seshadri. 2010. New policies for the stochastic inventory control problem with two supply sources. Operations Research 58(3) 734-745.

Sherbrooke, C.C. 1968. Metric: A multi-echelon technique for recoverable item control. Operations Research 16(1) $122-141$.

Sherbrooke, C.C. 2004. Optimal Inventory Modeling of Systems: Multi-Echelon Techniques. Kluwer Academic Publishers, Norwell, MA, USA.

Simon, R.M. 1971. Stationary properties of a two-echelon inventory model for low demand items. Operations Research 19(3) 761-773.

Sleptchenko, A., M.C. Van der Heijden, A. Van Harten. 2005. Using repair priorities to reduce stock investment in spare part networks. European Journal of Operational Research 163(3) 733-750.

Song, J.S., P. Zipkin. 2009. Inventories with multiple supply sources and networks of queues with overflow bypasses. Management Science 55(3) 362-372.

Sun, J., J.A. Van Mieghem. 2019. Robust dual sourcing inventory management: Optimality of capped dual index policies and smoothing. Manufacturing \& Service Operations Management 21(4) 912-931.

Svoboda, J., S. Minner, M Yao. 2019. Typology and literature review on multiple supplier inventory control models. Available at SSRN: http://ssrn.com/abstract=2995134.

Tiemessen, H.G.H., G.J.J.A.N. Van Houtum. 2013. Reducing costs of repairable inventory supply systems via dynamic scheduling. International Journal of Production Economics 143(2) 478-488.

Topan, E., Z.P. Bayındır, T. Tan. 2017. Heuristics for multi-item two-echelon spare parts inventory control subject to aggregate and individual service measures. European Journal of Operational Research 256(1) $126-138$.

Van Houtum, G.J.J.A.N., A.A. Kranenburg. 2015. Spare parts inventory control under system availability constraints, vol. 227. Springer. 
Veeraraghavan, S., A. Scheller-Wolf. 2008. Now or later: A simple policy for effective dual sourcing in capacitated systems. Operations Research 56(4) 850-864.

Whittemore, A.S., S.C. Saunders. 1977. Optimal inventory under stochastic demand with two supply options. SIAM Journal on Applied Mathematics 32(2) 293-305.

Wong, H., A.A. Kranenburg, G.J.J.A.N. Van Houtum, D. Cattrysse. 2007. Efficient heuristics for two-echelon spare parts inventory systems with an aggregate mean waiting time constraint per local warehouse. OR spectrum $\mathbf{2 9}(4) 699$. 


\section{Appendix A: Proofs}

Proof of Theorem 1 Let $S_{m, 0}$ and $T_{m}$ be fixed. Let $f: \mathbb{N}^{\left|N_{l}\right|} \rightarrow \mathbb{R}$ be the part of objective function (12) that depends on $S_{m, n}, n \in N_{l}$. Then, by omitting constants, objective function (12) reduces to

$$
f\left(S_{m, 1}, S_{m, 2}, \ldots, S_{m,\left|N_{l}\right|}\right)=\sum_{n \in N_{l}}\left[c_{a}^{m} S_{m, n}-p_{c} E B O_{m, n}\left(S_{m, n}\right)\right],
$$

where $E B O_{m, n}$ now depends only on $S_{m, n}$ because $S_{m, 0}$ and $T_{m}$ are fixed. By observing that each term in $f$ is precisely the cost of an independent Newsvendor type problem, one for each local warehouse $n \in N_{l}$, the desired result directly follows.

Proof of Lemma 1 Let $T_{m}$ be fixed and $S_{m, n}=0$ for all $n \in N_{l}$. Let $f: \mathbb{N} \rightarrow \mathbb{R}$ be the part of objective function (12) that depends on $S_{m, 0}$. Then, by omitting constants, objective function (12) reduces to

$$
f\left(S_{m, 0}\right)=c_{a}^{m} S_{m, 0}-p_{c} \sum_{n \in N_{l}} E B O_{m, n}\left(S_{m, 0}\right),
$$

where $E B O_{m, n}\left(S_{m, 0}\right)$ now depends only on $S_{m, 0}$ because $T_{m}$ is fixed and $S_{m, n}=0$ for all $n \in N_{l}$.

Recall that the number of parts outstanding at local warehouse $n \in N_{l}$ is the sum of the demand during transport and shipping time $t_{m, n}$ from the central warehouse to local warehouse $n, D_{m, n}$, and the number of backorders at the central warehouse that belong to local warehouse $n$. Hence, since $S_{m, n}=0$ for all $n \in N_{l}$, $E B O_{m, n}\left(S_{m, 0}\right)$ is equal to the sum of the expected backorders at the central warehouse that are from local warehouse $n$ and the expectation of $D_{m, n}$ (see Equation (2)).

Then, since the number of backorders at the central warehouse that belong to local warehouse $n$ is binomially distributed for a fixed total number of backorders (see Equation (1)), we have

$$
f\left(S_{m, 0}\right)=c_{a}^{m} S_{m, 0}-p_{c} \sum_{n \in N_{l}} \frac{\lambda_{m, n}}{\lambda_{m, 0}} E B O_{m, 0}\left(S_{m, 0}\right)=c_{a}^{m} S_{m, 0}-p_{c} E B O_{m, 0}\left(S_{m, 0}\right)
$$

where we have used the definition of $\lambda_{m, 0}$ and the fact that $\mathbb{E}\left[D_{m, n}\right]$ is constant and can thus be omitted. By observing that $f$ is precisely the cost of a Newsvendor type problem, the desired result directly follows.

Proof of Lemma 2 Let $T_{m}$ and $S_{m, 0}$ be fixed and take some local warehouse $n \in N_{l}$. Let $Y \geq_{s t} \tilde{Y}$ denote that a random variable $Y$ is stochastically larger than another random variable $\tilde{Y}$ in the usual stochastic order. Then, observe that $B O_{m, 0}\left(S_{m, 0}, T_{m}\right) \geq_{s t} B O_{m, 0}\left(S_{m, 0}+1, T_{m}\right)$. This implies that $B O_{m, 0}^{n}\left(S_{m, 0}, T_{m}\right) \geq_{s t}$ $B O_{m, 0}^{n}\left(S_{m, 0}+1, T_{m}\right)$, and thus $B O_{m, 0}^{n}\left(S_{m, 0}\right)+D_{m, n} \geq_{s t} B O_{m, 0}^{n}\left(S_{m, 0}+1\right)+D_{m, n}$, which is equivalent to $X_{m, n}\left(S_{m, 0}, T_{m}\right) \geq_{s t} X_{m, n}\left(S_{m, 0}+1, T_{m}\right)$. Hence, in particular it holds that $P\left\{X_{m, n}\left(S_{m, 0}, T_{m}\right) \leq x\right\} \leq$ $P\left\{X_{m, n}\left(S_{m, 0}+1, T_{m}\right) \leq x\right\}$ for any $x \in \mathbb{N}$. Hence, as $S_{m, n}^{*}\left(S_{m, 0}, T_{m}\right)$ is the smallest $S_{m, n}\left(S_{m, 0}, T_{m}\right)$ that satisfies Equation (14), we must have that $S_{m, n}^{*}\left(S_{m, 0}+1, T_{m}\right) \leq S_{m, n}^{*}\left(S_{m, 0}, T_{m}\right)$.

Proof of Theorem 2 This follows directly from Lemma 1 and Lemma 2.

\section{Appendix B: Pseudo-code of solution approaches}

This section provides pseudo-code of the DCG algorithm, including the exact solution method for $(S U B(m))$, as well as the two-step greedy heuristic. Note that in the pseudo-code of the exact solution method for $(S U B(m))$ (i.e. Algorithm 2), we continue the search over $T_{m}$ by examining 4 values beyond the last observed local minimum, that is $N^{\max }=4$. 

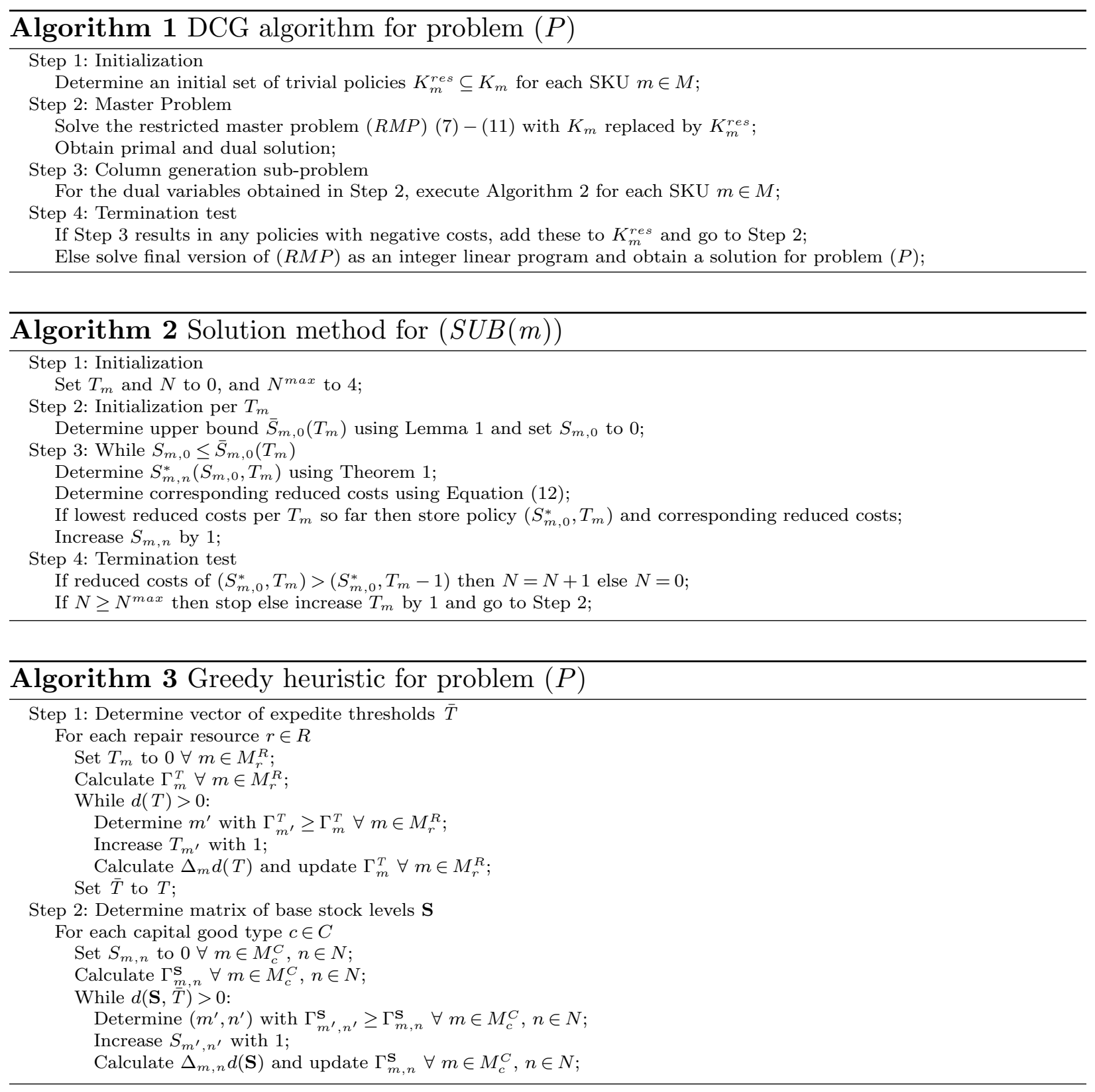

\section{Appendix C: Alternative formulation expediting repairs at additional costs}

Rather than minimizing a total cost rate, one might also be interested in minimizing a total initial cost consisting of both the total investment costs in spare parts (as in the original model) and the total expected discounted expediting costs. To this end, let $\beta>0$ denote the discounting factor. For a given vector of expedite thresholds $T$, the total expected discounted expediting costs over an infinite horizon is then given by:

$$
\tilde{C}_{e}(T)=\sum_{m \in M} \int_{0}^{\infty} e^{-\beta t} c_{e}^{m} \lambda_{m, 0} E X P_{m}\left(T_{m}\right) \mathrm{d} t=\sum_{m \in M} \frac{1}{\beta} c_{e}^{m} \lambda_{m, 0} E X P_{m}\left(T_{m}\right),
$$

where the second equality follows from assuming that the system starts in steady state. The remaining analysis is now identical to the case with a total cost rate, with $C_{e}(T)$ changed to $\tilde{C}_{e}(T)$. 


\section{Appendix D: Allowing for commonality}

In this section, we relax the assumption that $M_{c}^{C}$ and $M_{r}^{R}$ partition $M$, that is, the assumption that each SKU $m \in M$ occurs in the configuration of only one capital good type $c \in C$ and uses only one resource $r \in R$ for its repair. We first introduce additional notation to differentiate between demands for the same SKU that stem from different capital good types. We then briefly describe how problem $(P)$ and its solution approaches change when commonality between SKUs is allowed.

Let $\lambda_{m, n, c}$ denote the demand intensity for SKU $m \in M$ at warehouse $n \in N$ originating from capital good type $c \in C$. If SKU $m$ does not occur in the configuration of capital good type $c$, then $\lambda_{m, n, c}=0$ by definition. Let the fraction of demands for SKU $m$ at warehouse $n$ that originate from capital good type $c$ over all demands for that SKU at that warehouse be denoted by $\delta_{m, n}^{c}=\frac{\lambda_{m, n, c}}{\sum_{k \in C} \lambda_{m, n, k}}$.

The aggregate mean number of backorders for each capital good type $c \in C$ is now given by a weighted sum of the mean number of backorders for all SKUs occurring in the configuration of capital good type $c$, with the fractions $\delta_{m, n}^{c}$ as weights. That is,

$$
E B O_{c}(\mathbf{S}, T)=\sum_{m \in M_{c}^{C}} \sum_{n \in N_{l}} \delta_{m, n}^{c} E B O_{m, n}\left(\mathbf{S}_{m}, T_{m}\right) .
$$

The definition of the aggregate mean fraction of failed parts that are expedited per repair resource $r \in R$, i.e. $\operatorname{EXP}_{r}(T)$, remains however the same: SKUs now simply contribute to multiple aggregate mean fractions of expedited repairs whenever they require multiple resources for their repair. Hence, with $E B O_{c}(\mathbf{S}, T)$ now being defined as in Equation (19), we readily generalize our decision problem to the setting where commonality between SKUs is allowed.

The rest of the analysis goes along similar lines as for the setting without commonality. In particular, constraint (8) in the master problem of the DCG algorithm should be reformulated to

$$
\sum_{m \in M_{c}^{C}} \sum_{n \in N_{l}} \sum_{k \in K_{m}} \delta_{m, n}^{c} E B O_{m, n}\left(\mathbf{S}_{m}^{k}, T_{m}^{k}\right) x_{m}^{k} \leq \mathcal{B}_{c}^{\max }, \quad \forall c \in C,
$$

which now incorporates our new definition for the aggregate mean number of backorders.

As SKUs may now belong to multiple capital good types and may now use multiple resources for their repair, the column generation sub-problem for SKU $m \in M$ is now formulated as follows:

$$
\begin{array}{rc}
\widetilde{S U B}(m)) \min _{\left\{\left(\mathbf{S}_{m}, T_{m}\right)\right\}} & \sum_{n \in N} c_{a}^{m} S_{m, n}-\sum_{c \in C} \sum_{n \in N_{l}} p_{c} \delta_{m, n}^{c} E B O_{m, n}\left(\mathbf{S}_{m}, T_{m}\right)-\sum_{r \in R} \rho_{r} \delta_{m}^{r} E X P_{m}\left(T_{m}\right)-v_{m} \\
\text { subject to } & \mathbf{S}_{m} \in \mathbb{N}_{0}^{|N|}, \quad T_{m} \in n \in \mathbb{N}_{0} .
\end{array}
$$

Note that the structure of $(\widetilde{S U B}(m))$ is identical to $(S U B(m))$. It is therefore readily verified that all properties as well as the exact solution method presented in Section 5.2 also hold for $(\widetilde{S U B}(m))$, with the critical fraction $\frac{p_{c}+c_{a}^{m}}{p_{c}}$ in Theorem 1 and Lemma 1 changed to $\frac{\sum_{c \in C} \delta_{m, n}^{c} p_{c}+c_{a}^{m}}{\sum_{c \in C} \delta_{m, n}^{c} p_{c}}$.

In addition to the DCG algorithm, the two-step greedy approach can also be applied almost immediately to the setting where commonality between SKUs is allowed. The only difference is that the decreases in distances $\Delta_{m} d(T)$ and $\Delta_{m, n} d(\mathbf{S}, \bar{T})$ should now be calculated over multiple repair resources and multiple capital good types, respectively, with the additional note that in calculating $\Delta_{m, n} d(\mathbf{S}, \bar{T})$, we use Equation (19) for the aggregate mean number of backorders for each capital good type $c \in C$. 


\section{Appendix E: Numerical experiments}

In this section, we report on our numerical experiments. The main objective of these experiments is to examine how the performance of our solution approaches, in terms of $\% G A P$ and $\% R E D$, is affected by the input parameters of the decision problem. To this end, we consider a large test bed of randomly generated instances based on data representative for the capital goods industry. We first do so for the original decision problem, i.e. problem $(P)$, and we subsequently treat the alternate setting in which additional costs are associated with expediting as well, i.e. problem $(\widehat{P})$.

\section{E.1. Numerical experiments involving problem $(P)$}

The test bed for the numerical experiments of this section consists of 2592 instances obtained through all combinations of the parameter values in Table 6. For each instance, we first use an uniform distribution $U[0.005,0.25]$ to generate the demand intensity for each SKU $m \in M$ at all local warehouses $n \in N_{l}$, and subsequently multiply this generated demand intensity at each local warehouse $n \in N_{l}$ with a demand intensity multiplier, denoted $\ell$. We consider symmetric instances in which demand intensities are identical across all local warehouses but varied for different SKUs, i.e. $\ell=1$, as well as asymmetric cases in which demand intensities are varied across both local warehouses and different SKUs, i.e. $\ell=U[0.5,1.5]$. Note that in each instance, we assign all SKUs uniformly at random to a repair resource set $M_{r}^{R}$ for $r=1, \ldots,|R|$.

Table 6 Input parameter values for test bed involving problem $(P)$

\begin{tabular}{|c|c|c|c|}
\hline & Input parameter & No. of choices & Values \\
\hline 1 & Number of local warehouses, $\left|N_{l}\right|$ & 3 & $2,4,6$ \\
\hline 2 & Number of capital good types, $|C|$ & 2 & 2,4 \\
\hline 3 & Number of repair resources, $|R|$ & 2 & 2,4 \\
\hline 4 & Number of SKUs per capital good type, $\left|M_{c}^{C}\right|$ & 3 & $20,50,100$ \\
\hline 5 & $\begin{array}{l}\text { Lead time from the central warehouse to local warehouse } n \in N_{l} \\
\text { of SKU } m \in M, t_{m, n}\end{array}$ & 1 & 1 \\
\hline 6 & Expedited repair lead time of SKU $m \in M, t_{m, 0}^{2}$ & 2 & 1,2 \\
\hline 7 & Additional regular repair lead time of SKU $m \in M, t_{m, 0}^{1}$ & 2 & 3,5 \\
\hline 8 & Acquisition cost of SKU $m \in M, c_{a}^{m}$ & 1 & $U[100,1000]$ \\
\hline 9 & $\begin{array}{l}\text { Demand intensity for SKU } m \in M \text { at each local warehouse } n \in \\
N_{l}, \lambda_{m, n}\end{array}$ & 1 & $U[0.005,0.25]$ \\
\hline 10 & Demand intensity multiplier, $\ell$ & 2 & $1, \mathrm{U}[0.5,1.5]$ \\
\hline 11 & $\begin{array}{l}\text { Maximally allowed mean number of backorders over all SKUs } \\
m \in M_{c}^{C} \text { for capital good type } c \in C, \mathcal{B}_{c}^{\max }\end{array}$ & 3 & $\begin{array}{l}\nu \sum_{m \in M_{C}^{C}}^{C} \sum_{n \in N_{l}} \lambda_{m, n} \\
\text { for } \nu=0.04,0.06,0.08\end{array}$ \\
\hline 12 & $\begin{array}{l}\text { Maximally allowed mean fraction of expedited repairs over all } \\
\text { SKUs } m \in M_{r}^{R} \text { that use repair resource } r \in R \text { during their } \\
\text { repair, } \mathcal{E}_{r}^{\max }\end{array}$ & 3 & $0.05,0.10,0.20$ \\
\hline
\end{tabular}

The results of our numerical experiments involving problem $(P)$ are summarized in Table 7 . In this table, we present the average and maximum $\%$ GAP and computation times (in seconds) of both solution approaches as well as the average and maximum $\% R E D$. We first distinguish between subsets of instances with the same value for a specific input parameter of Table 6 and then present the results for all instances. That is, the bottom row of Table 7 contains the results computed over all instances, and the other rows present the results computed over subsets of instances that have the same value for a specific input parameter.

The main observations drawn from Table 7 can be summarized as follows:

- The DCG algorithm performs very well. The average and maximum \%GAP are 0.27 and 0.77 percent, respectively. 
Drent and Arts: Expediting in Two-Echelon Spare Parts Inventory Systems

Table 7 Summary of numerical results involving problem $(P)$

\begin{tabular}{|c|c|c|c|c|c|c|c|c|c|c|c|}
\hline \multirow[b]{3}{*}{ Input parameter } & \multirow[b]{3}{*}{ Value } & \multicolumn{4}{|c|}{ DCG algorithm } & \multicolumn{4}{|c|}{ Greedy heuristic } & \multirow{2}{*}{\multicolumn{2}{|c|}{$\frac{\text { Benchmark }}{\% R E D}$}} \\
\hline & & \multicolumn{2}{|c|}{$\% G A P$} & \multicolumn{2}{|c|}{ CPU time $(s)$} & \multicolumn{2}{|c|}{$\% G A P$} & \multicolumn{2}{|c|}{ CPU time $(s)$} & & \\
\hline & & Avg & $\operatorname{Max}$ & Avg & $\operatorname{Max}$ & Avg & $\operatorname{Max}$ & Avg & $\operatorname{Max}$ & Avg & $\operatorname{Max}$ \\
\hline \multirow{3}{*}{$\begin{array}{l}\text { Number of local } \\
\text { warehouses, }\left|N_{l}\right|\end{array}$} & 2 & 0.32 & 0.77 & 10.75 & 71.39 & 3.07 & 8.43 & 0.24 & 0.77 & 9.66 & 19.61 \\
\hline & 4 & 0.26 & 0.63 & 61.38 & 292.86 & 2.23 & 5.75 & 1.23 & 4.42 & 7.54 & 14.70 \\
\hline & 6 & 0.23 & 0.55 & 230.01 & 1271.85 & 1.85 & 4.77 & 3.49 & 12.85 & 6.62 & 13.27 \\
\hline \multirow{2}{*}{$\begin{array}{l}\text { Number of capital good } \\
\text { types, }|C|\end{array}$} & 2 & 0.29 & 0.77 & 62.42 & 611.31 & 2.36 & 8.43 & 1.10 & 6.49 & 7.86 & 19.61 \\
\hline & 4 & 0.26 & 0.56 & 139.00 & 1271.85 & 2.41 & 8.04 & 2.20 & 12.85 & 8.01 & 18.63 \\
\hline \multirow{2}{*}{$\begin{array}{l}\text { Number of repair } \\
\text { resources, }|R|\end{array}$} & 2 & 0.25 & 0.57 & 100.54 & 1271.85 & 2.40 & 8.43 & 1.65 & 12.85 & 8.02 & 19.61 \\
\hline & 4 & 0.29 & 0.77 & 100.89 & 1135.98 & 2.37 & 8.01 & 1.65 & 12.78 & 7.85 & 18.32 \\
\hline \multirow{3}{*}{$\begin{array}{l}\text { Number of SKUs per } \\
\text { capital good type, }\left|M_{c}^{C}\right|\end{array}$} & 20 & 0.36 & 0.77 & 35.37 & 328.09 & 2.49 & 8.43 & 0.58 & 2.70 & 7.85 & 18.81 \\
\hline & 50 & 0.24 & 0.51 & 88.36 & 731.60 & 2.34 & 7.64 & 1.45 & 6.43 & 8.01 & 19.61 \\
\hline & 100 & 0.21 & 0.50 & 178.42 & 1271.85 & 2.32 & 6.52 & 2.92 & 12.85 & 7.96 & 18.49 \\
\hline \multirow{2}{*}{$\begin{array}{l}\text { Expedited repair lead } \\
\text { time, } t_{m, 0}^{2}\end{array}$} & 1 & 0.28 & 0.77 & 79.87 & 926.74 & 2.57 & 8.43 & 1.43 & 9.99 & 8.98 & 19.61 \\
\hline & 2 & 0.26 & 0.75 & 121.55 & 1271.85 & 2.20 & 7.27 & 1.88 & 12.85 & 6.89 & 14.91 \\
\hline \multirow{2}{*}{$\begin{array}{l}\text { Additional regular repair } \\
\text { lead time, } t_{m, 0}^{1}\end{array}$} & 3 & 0.28 & 0.77 & 67.21 & 682.63 & 2.48 & 8.43 & 1.38 & 9.39 & 6.38 & 14.24 \\
\hline & 5 & 0.27 & 0.75 & 134.22 & 1271.85 & 2.28 & 6.70 & 1.92 & 12.85 & 9.50 & 19.61 \\
\hline \multirow{2}{*}{$\begin{array}{l}\text { Demand intensity } \\
\text { multiplier, } \ell\end{array}$} & 1 & 0.28 & 0.77 & 111.34 & 1271.85 & 3.69 & 8.43 & 1.75 & 12.85 & 7.92 & 19.61 \\
\hline & $\mathrm{U}[0.5,1.5]$ & 0.26 & 0.75 & 90.08 & 939.03 & 1.07 & 3.15 & 1.55 & 11.47 & 7.95 & 18.81 \\
\hline \multirow{3}{*}{$\begin{array}{l}\text { Fraction of total demand } \\
\text { that may be backordered, } \\
\nu\end{array}$} & 0.04 & 0.27 & 0.75 & 100.48 & 1104.62 & 2.43 & 8.43 & 1.74 & 12.85 & 7.98 & 18.37 \\
\hline & 0.06 & 0.27 & 0.66 & 100.49 & 992.75 & 2.40 & 7.64 & 1.65 & 11.72 & 7.87 & 19.61 \\
\hline & 0.08 & 0.28 & 0.77 & 101.17 & 1271.85 & 2.32 & 8.04 & 1.57 & 11.69 & 7.97 & 18.63 \\
\hline \multirow{3}{*}{$\begin{array}{l}\text { Fraction of total demand } \\
\text { that may be expedited, } \\
\mathcal{E}_{r}^{\max }\end{array}$} & 0.05 & 0.27 & 0.62 & 104.42 & 1271.85 & 2.13 & 6.09 & 1.70 & 12.85 & 5.21 & 9.83 \\
\hline & 0.1 & 0.28 & 0.69 & 98.99 & 1135.98 & 2.29 & 8.01 & 1.66 & 12.43 & 7.79 & 14.81 \\
\hline & 0.2 & 0.27 & 0.77 & 98.72 & 1125.12 & 2.73 & 8.43 & 1.59 & 11.88 & 10.81 & 19.61 \\
\hline \multicolumn{2}{|l|}{ Total } & 0.27 & 0.77 & 100.71 & 1271.85 & 2.38 & 8.43 & 1.66 & 12.85 & 7.94 & 19.61 \\
\hline
\end{tabular}

- The greedy heuristic performs very well when demand intensities are asymmetric. The average and maximum \%GAP in this case are only 1.07 and 3.15, respectively. The greedy heuristic performs slightly worse with symmetric demand intensities: The average $\%$ GAP is 3.69 but instances with 8 or more do occur. This observation is in line with previous research which examined greedy heuristics in multi-item spare parts problems (e.g., Topan et al. 2017). A possible explanation for this slightly worse performance is due to how the second step of the greedy heuristic works. With symmetric demand intensities, we have the property that if in a given iteration the base stock level of a specific SKU is increased at one local warehouse, then also the base stock levels of the same SKU at all other local warehouses are most likely increased in the succeeding iterations. However, in most practical situations in which each local warehouse serves a distinct market with a different demand structure, one will most likely encounter asymmetric demand intensities and hardly ever symmetric demand intensities.

- The average \%GAP of both solution approaches seem to decrease as the instance size (in terms of the number of local warehouses, capital good types and SKUs per capital good type) becomes larger. This is very convenient since we typically face large-sized instances in practice. The average \%GAP percent of the DCG algorithm tends to increase with the number of repair resources. This is not surprising, because problem $(M P)$ has $|M|+|C|+|R|$ constraints and the same number of basic variables in an optimal solution. Since constraint (10) assures that for each SKU $m \in M$ a convex combination of policies is chosen, there is a basic variable for each SKU $m$. Hence, there are at most $|C|+|R|$ SKUs for which the optimal solution 
to problem $(M P)$ is fractional. This explains why the $G A P$ percent increases with the number of repair resources. Note that this does not hold for the number of capital good types because the number of basic variables that increase with the number of capital good types is clearly more than the corresponding increase in the maximum number of SKUs for which the optimal solution to problem $(M P)$ is fractional.

- The average \% GAP of the DCG algorithm tends to decrease as the fraction of total demand that may be expedited or backordered decreases. This also seems to hold for the greedy heuristic, except with symmetric demand intensities. In the latter case, the average $\% G A P$ of the greedy heuristic increases when the fraction of total demand that may be backordered decreases.

- The greedy heuristic is the most efficient heuristic in terms of computation time. The computation time of the DCG algorithm is considerably higher. Over 98 percent of that computation time is spent on solving the sub-problems. This task can also be parallelized using a multi-threaded approach, which would reduce the computation time of the DCG algorithm even further. The computation time of both solution approaches increases as the problem size (in terms of the number of local warehouses, capital good types and SKUs per capital good type) gets larger and decreases when the means of the repair lead times get smaller.

- The stock investment reductions that can be achieved because of the possibility to expedite the repair of parts in short supply are quite high with an average stock investment reduction of around 7.9 percent and even reductions of up to 19.61 percent.

- The stock investment reductions due to our dynamic repair policy increase when the additional regular repair lead time or the fraction of total demand that may be expedited increase, and decrease when the expedited repair lead time increase.

\section{E.2. Numerical experiments involving problem $(\widehat{P})$}

In this section, we consider a test bed that is identical to the test bed used in the previous section except for the two input parameters that are specific to problem $(P)$, i.e. $\mathcal{E}_{r}^{\max }$ and $|R|$. We replace those two input parameters with the two input parameters that are specific to problem $(\widehat{P})$, i.e. the expediting cost multiplier $\kappa$ and the useful life span $\tau$. We vary the expediting cost multiplier over three levels: $\kappa \in\{0.002,0.004,0.006\}$. Assuming that a regular repairs costs 5 to 10 percent of the acquisition cost of a part, these expediting cost multipliers imply a relative cost premium for expedited repairs of 2 to 12 percent. Such relative cost premiums are often reported in the dual-sourcing literature, where a cost premium is paid to utilize an expedited supplier over a regular supplier (e.g., Klosterhalfen et al. 2011, Sun and Van Mieghem 2019). The useful lifespan (in years) is varied over two levels, both of which are representative for the capital goods industry: $\tau \in\{8,10\}$ (we now explicitly assume that demand intensities are per day). Note that the total number of instances remains unchanged and hence this test bed also consists of 2592 instances.

Table 8 summarizes the results of our numerical experiments involving problem $(\widehat{P})$. We present the average and maximum \%GAP and computation times (in seconds) of the DCG algorithm, the average and maximum \%RED as well as the average and maximum EXP (expressed as percentages), i.e. the aggregated mean fraction of failed parts that receive expedited repair. Similar to Table 7 in the previous section, we first distinguish between subsets of instances with the same value for a specific input parameter and then present the results for all instances. 
Table 8 Summary of numerical results involving problem $(\widehat{P})$

\begin{tabular}{|c|c|c|c|c|c|c|c|c|c|}
\hline \multirow[b]{3}{*}{ Input parameter } & \multirow[b]{3}{*}{ Value } & \multicolumn{4}{|c|}{ DCG algorithm } & \multirow{2}{*}{\multicolumn{2}{|c|}{$\frac{\text { Benchmark }}{\% R E D}$}} & \multirow{2}{*}{\multicolumn{2}{|c|}{$\frac{\text { Expediting }}{\operatorname{EXP}(\%)}$}} \\
\hline & & \multicolumn{2}{|c|}{$\% G A P$} & \multicolumn{2}{|c|}{ CPU time $(s)$} & & & & \\
\hline & & Avg & Max & Avg & Max & Avg & Max & Avg & Max \\
\hline \multirow{3}{*}{$\begin{array}{l}\text { Number of local } \\
\text { warehouses, }\left|N_{l}\right|\end{array}$} & 2 & 0.22 & 0.54 & 11.69 & 75.27 & 7.59 & 29.29 & 23.13 & 94.02 \\
\hline & 4 & 0.18 & 0.51 & 95.15 & 676.21 & 4.10 & 18.44 & 16.18 & 84.33 \\
\hline & 6 & 0.15 & 0.52 & 421.78 & 2757.51 & 2.88 & 14.05 & 12.81 & 72.81 \\
\hline \multirow{2}{*}{$\begin{array}{l}\text { Number of capital good } \\
\text { types, }|C|\end{array}$} & 2 & 0.15 & 0.54 & 111.81 & 1767.52 & 4.89 & 28.98 & 17.24 & 94.02 \\
\hline & 4 & 0.21 & 0.52 & 240.61 & 2757.51 & 4.82 & 29.29 & 17.51 & 93.64 \\
\hline \multirow{2}{*}{ Useful lifespan (years), $\tau$} & 8 & 0.19 & 0.54 & 161.15 & 2757.51 & 5.74 & 29.29 & 21.93 & 94.02 \\
\hline & 10 & 0.17 & 0.52 & 191.26 & 2587.05 & 3.97 & 21.11 & 12.81 & 65.99 \\
\hline \multirow{3}{*}{$\begin{array}{l}\text { Number of SKUs per } \\
\text { capital good type, }\left|M_{c}^{C}\right|\end{array}$} & 20 & 0.22 & 0.54 & 59.22 & 661.44 & 4.82 & 29.29 & 17.55 & 93.85 \\
\hline & 50 & 0.16 & 0.52 & 151.53 & 1515.09 & 4.87 & 28.98 & 17.34 & 94.02 \\
\hline & 100 & 0.16 & 0.50 & 317.87 & 2757.51 & 4.87 & 28.81 & 17.24 & 92.57 \\
\hline \multirow{2}{*}{$\begin{array}{l}\text { Expedited repair lead } \\
\text { time, } t_{m, 0}^{2}\end{array}$} & 1 & 0.19 & 0.54 & 135.44 & 2499.62 & 5.70 & 29.29 & 18.45 & 94.02 \\
\hline & 2 & 0.17 & 0.52 & 216.97 & 2757.51 & 4.01 & 22.08 & 16.29 & 93.24 \\
\hline \multirow{2}{*}{$\begin{array}{l}\text { Additional regular repair } \\
\text { lead time, } t_{m, 0}^{1}\end{array}$} & 3 & 0.17 & 0.54 & 129.29 & 1922.82 & 2.38 & 12.07 & 9.05 & 43.07 \\
\hline & 5 & 0.19 & 0.52 & 223.13 & 2757.51 & 7.33 & 29.29 & 25.70 & 94.02 \\
\hline \multirow{2}{*}{$\begin{array}{l}\text { Demand intensity } \\
\text { multiplier, } \ell\end{array}$} & 1 & 0.19 & 0.54 & 191.42 & 2757.51 & 4.75 & 28.49 & 16.96 & 94.02 \\
\hline & $\mathrm{U}[0.5,1.5]$ & 0.16 & 0.51 & 160.99 & 2300.56 & 4.96 & 29.29 & 17.78 & 93.64 \\
\hline \multirow{3}{*}{$\begin{array}{l}\text { Fraction of total demand } \\
\text { that may be backordered, } \\
\nu\end{array}$} & 0.04 & 0.18 & 0.52 & 173.86 & 2757.51 & 5.10 & 29.29 & 17.77 & 93.24 \\
\hline & 0.06 & 0.18 & 0.52 & 178.50 & 2458.69 & 4.84 & 28.59 & 17.41 & 94.02 \\
\hline & 0.08 & 0.18 & 0.54 & 176.26 & 2587.05 & 4.63 & 29.23 & 16.94 & 93.85 \\
\hline \multirow{3}{*}{$\begin{array}{l}\text { Expediting cost multiplier, } \\
\kappa\end{array}$} & 0.002 & 0.19 & 0.54 & 93.84 & 1081.79 & 9.68 & 29.29 & 40.13 & 94.02 \\
\hline & 0.004 & 0.19 & 0.52 & 188.04 & 2304.80 & 3.26 & 12.83 & 8.45 & 26.59 \\
\hline & 0.006 & 0.16 & 0.51 & 246.74 & 2757.51 & 1.62 & 7.64 & 3.54 & 12.59 \\
\hline \multicolumn{2}{|l|}{ Total } & 0.18 & 0.54 & 176.21 & 2757.51 & 4.86 & 29.29 & 17.37 & 94.02 \\
\hline
\end{tabular}

The numerical results in Table 8 are mostly in line with the numerical results of the previous section. We will now briefly discuss the main differences and important observations:

- The DCG algorithm also yields excellent results when it is applied to problem $(\widehat{P})$ : The average and maximum \%GAP are 0.18 and 0.54 percent, respectively. These optimality gaps are smaller than the optimality gaps of the DCG algorithm applied to problem $(P)$, which is not surprising as problem $(\widehat{P})$ has $|R|$ fewer constraints than problem $(P)$ and therefore fewer SKUs for which the optimal solution to problem $(\widehat{M P})$ is fractional (see also our discussion related to the optimality gaps in the previous section).

- Although the computation time of the DCG algorithm is higher for problem $(\widehat{P})$ than for problem $(P)$, it is still well within acceptable bounds given the size and strategic nature of the decision problem. We note that this increase in computation time can in large part be explained by the fact that we use the two-step greedy heuristic to create initial policies for the DCG algorithm; this heuristic is not devised specifically for problem $(\widehat{P})$ and these initial policies are therefore not necessarily good. This in contrast with problem $(P)$ where the two-step greedy heuristic leads to very good policies for initializing the DCG algorithm.

- The possibility to expedite the repair of failed parts at additional costs is effective in reducing the total cost rate with average reductions of around 4.9 percent and even reductions up to 29.29 percent. Hence, anticipating expediting decisions that will be made later with investment decisions in repairable spare parts leads to substantial savings, even when those expedited repairs come at an additional price.

- The reductions in the total cost rate due to our dynamic repair policy decrease when expediting repairs becomes more costly, and increase when the useful lifespan of SKUs becomes shorter. 


\section{Appendix F: Approach to determine benchmark for case study}

Our benchmark for the case study is the current solution that NS uses. In this solution, the investment in each spare part is determined by the stocking model of Servigistics. This stocking model is a single-item model that essentially ensures that for each SKU $m \in M$ sufficiently many spare parts are acquired to cover the lead time demand plus a safety level to protect against variability in demand. Hence, the amount of stock of SKU $m \in M$ determined by this stocking model is given by:

$$
S_{m}^{n s}=\mu+k \cdot \sigma,
$$

where $k$ is a safety factor, and where $\mu$ and $\sigma$ are the average and standard deviation of the demand over the total lead time (regular repair lead time + transportation time). The safety factor $k$ for NS is quite involved but is set such that the fill rate for each SKU is $98 \%$. See also Section 7.2 of Drent (2017) for more details.

Given $S_{m}^{n s}$, we determine the best achievable availability performance by optimizing expediting decisions and stock placement within our modeling framework. That is, we want to determine an expedite threshold $\tilde{T}_{m} \in \mathbb{N}_{0}$ and a base stock levels vector $\tilde{\mathbf{S}}_{m} \in\left\{\mathbb{N}_{0}^{|N|}: \mathbf{1} \cdot \mathbb{N}_{0}^{|N|}=S_{m}^{n s}\right\}$ such that $E B O(\tilde{\mathbf{S}}, \tilde{T})$ is minimized while $E X P_{\text {mechanical }}(\tilde{T}) \leq 0.3$ and $E X P_{\text {electronic }}(\tilde{T}) \leq 0.3$. This results in the following mathematical formulation of the optimization problem:

$$
\begin{array}{ll}
\min _{\{\tilde{\mathbf{S}}, \tilde{T}\}} & E B O(\tilde{\mathbf{S}}, \tilde{T}) \\
\text { subject to } & E X P_{\text {mechanical }}(\tilde{T}) \leq 0.3, \\
& \operatorname{EXP}_{\text {electronic }}(\tilde{T}) \leq 0.3, \\
& \tilde{\mathbf{S}} \in \mathscr{C}, \quad \tilde{T} \in \mathbb{N}_{0}^{|M|},
\end{array}
$$

where $\mathscr{C}=\left\{\tilde{\mathbf{S}}: \mathbf{1} \cdot \tilde{\mathbf{S}}_{m}=S_{m}^{n s} \forall m \in M\right\}$. We solve problem $(N S)$ using a decomposition and column generation approach similar to our approach to solve problem $(P)$, described in Section 5. The resulting corresponding sub-problem, however, does not allow for an easy solution method other than enumeration over $\tilde{T}_{m}$, and for each $\tilde{T}_{m}$, enumerating over all possible allocations of $\tilde{\mathbf{S}}_{m}$ over all local warehouses; see Section 7.2 of Drent (2017) for further details. 